\title{
Feeding a Low-protein Maternal Diet Affects Qinghai Bamei Piglet Jejunal Microbiome-metabolome Response
}

\section{Jipeng Jin}

Qinghai University

\section{Liping Zhang}

Gansu Agricultural University

\section{Qian Chen}

Qinghai University

\section{Cunming Ma}

Qinghai Province Huzhu County Bamei Pig Seed Breeding Farm

Jianlei Jia ( $\square$ jiajianlei87@163.com )

Qinghai University

\section{David. P. Casper}

Casper's Calf Ranch

\section{Research Article}

Keywords: Bamei suckling piglets, 16S rRNA, metabolic profiles, low-protein diet, Bioinformatics

Posted Date: June 28th, 2021

DOl: https://doi.org/10.21203/rs.3.rs-591216/v1

License: (1) This work is licensed under a Creative Commons Attribution 4.0 International License. Read Full License 


\section{Abstract}

This experiment investigated the impacts of feeding a maternal low-CP concentration diet having isoessential amino acids on new born suckling piglets intestinal microbial composition and metabolic profiles. The Bamei swine breed was selected due to high meat quality and flavor, but demonstrates slower growth rates which may be related to jejunal nutrient supply. Forty randomly selected purebred Bamei sows were divided into two groups and fed a low dietary CP $(12 \%, \mathrm{LP})$ or a normal CP $(14 \%, \mathrm{CON})$ diet, respectively, but formulated to contain similar (iso-) essential amino acid concentrations per current recommendations. At 21 days, 12 piglets were randomly selected from each treatment and euthanized with jejunum content samples collected. The 16S rRNA gene sequencing and mass spectrometry-based metabolomics profiling were combined as an integrated approach for evaluating the functional impact of maternal CP concentrations on piglet intestinal microbiome. Even though piglets demonstrated similar 0 to $21 \mathrm{~d}$ ADG among treatments, the jejunum relative weight, villus width, crypt depth and muscular thickness were increased $(P<0.05)$, while villus height, and villus height:crypt depth were reduced $(P<$ $0.05)$ for the material LP compared to the maternal fed CON diet. Maternal CP concentrations can modify the intestinal microbial composition of Bamei suckling piglets. The relative abundances of the bacterial species Escherichia-Shigella, Actinobacillus, Clostridium_sensu_stricto_1, Veillonella, and Turicibacter were increased $(P<0.05)$ in the maternal LP fed diet compared with the maternal fed CON diet. Jejunal digesta metabolomics analysis indicated that several amino acids were metabolized (i.e. cys, met, tyr phe and trp), biosynthesized (arg phe, tyr, and trp), or degraded (lys) were enriched $(P<0.05)$ for the maternal fed LP compared with the maternal fed CON. Correlation analysis demonstrated that certain intestinal bacterial genera were highly related to the histomorphology and altered intestinal microbiota metabolites. In conclusion, maternal dietary CP concentrations in excess of protein and amino acid requirements not only altered suckling Bamei piglets histomorphology, microbial composition and function, but also modulated jejunum microbial metabolic profiles, which aids in understanding the beneficial effects when feeding a maternal LP diet on piglet intestinal health.

\section{Introduction}

The Bamei swine breed is a local swine breed in the Qinghai Province of the People's Republic of China. Bamei pigs are known for their meat quality and flavor, but are known to be slow growing (Jin, 2006; Yang and Gun, 2007). The Qinghai plateau has used both natural and artificial selection practices for developing Bamei pigs developing a strong adaptability to the plateau, fat deposition, and good meat quality characteristics. However, Bamei's slow growth rates combined with the plateau's low feed quality/digestibility are important constraints limiting the Qinghai's growth potential of the Bamei swine industry. This results in international implications for meat imports along with grains and protein sources that need to be addressed for food production. Bamei adipose growth rates increase dramatically after 35 $\mathrm{kg}$, while muscle growth rates decrease dramatically after achieving $55 \mathrm{~kg}$ (Yang and Song, 1991). China's continuous improvement of people's living standards has resulted in Bamei pork becoming more popular. 
The gastrointestinal tract's microbial ecosystem is dynamic and complex with the composition known to vary widely across healthy individuals (Huttenhower et al., 2012). In the human and animal gastrointestinal tract lives a large and diverse microbial community playing a vital role in host health (Kuang et al., 2019), muscosal immunological environment maturation (Pattaroni et al., 2018), precision medicine development (Kuntz and Gilbert, 2017), and assisting with intestinal barrier integrity (MartinezLopez et al., 2019). Over the last decade, numerous studies have reported that the intestinal microbiome composition plays an important role in regulating the metabolic health of both rodents and humans (Kreznar et al., 2017). Recent rodent work suggests the major dietary factors regulating intestinal microbiome taxonomic composition are protein and carbohydrate intake (Holmes et al., 2017).

The intestinal microbiome is a complex and dynamic ecosystem of bacterial species being in a continual state of flux and highly susceptible to numerous environmental factors, especially dietary nutrient supply. Reducing CP by 2 to 4 percentage units by adding crystalline amino acids (AA) to meet NRC (2012) nutrient recommendations has increased nitrogen utilization, reduced feed costs and nitrogen excretion, while promoting intestinal health and meat quality with similar growth performance (Wang et al., 2018). The high-quality protein source shortage is a global problem, but especially for China's large population. Since 2002, the world's largest soybean importer is China (Zhang and Reed, 2008). In 2016, China imported 8.391 billion tons soybeans, which is $>26 \%$ of global production. Decreasing dietary protein concentrations can effectively reduce pressure on protein source availability (Wang et al., 2018). Many studies demonstrate dietary $\mathrm{CP}$ concentrations versus $\mathrm{CP}$ source, have a greater impact on intestinal microbiota composition (Rist et al., 2013). Previous studies have focused on changes in large intestinal microbiota, while ignoring the bacteria's role for the small intestine (Dai et al., 2010). Moderate diet protein restriction may alter intestinal microbiota composition while improving adult pig ileal barrier function (Fan et al., 2017). Chen et al. (2018) reported that decreasing dietary CP concentration $3 \%$ units reduced ileal Streptococcus spp., while increasing Lactobacillus spp. and Bifidobacterium spp. These ileal microbiota alterations improved intestinal stem cell proliferation and altered tight junction protein distribution resulting in similar intestinal barrier function. Therefore, feeding dietary LP concentrations has advanced while maintaining essential amino acid supply and has been applied to swine production. The application of 16S rRNA high-throughput sequencing technology provides methods for determining if maternal dietary CP concentration can alter intestinal microbial composition at different physiological stages and intestinal locations.

The Bamei pig was selected due to its popularity with local consumers, but it is a slower growing swine breed, which needs to be addressed. He et al. (2016) reported that IGF-1, insulin, leptin and amino acids may be associated with slow growth. The hypothesis was that altering maternal dietary CP concentrations would alter the intestinal microbiota and metabolites for the suckling piglets. The $16 \mathrm{~S}$ rRNA gene sequencing method was integrated with LC-MS metabolomics to analyze maternal dietary LP concentration on piglet intestinal microbiome and metabolite profiles. The relationships between metabolites and microbiota were explored as well.

\section{Material And Methods}




\subsection{Ethics Statement}

The use of animals was kept to an absolute minimum required to achieve statistical significance for validation purposes; a total of 40 animals were used for the work described in this paper. All procedures involving the Huzhu Bamei sows were conducted at the Qinghai Province Huzhu County Bamei Pig Seed Breeding Farm (Huzhu, China). All procedures were conducted in accordance with China animal welfare law Act 2011, approved by institutional ethical review committees (the State Key Laboratory of Plateau Ecology and Agriculture, Qinghai University Animal Ethics Committee)and conducted under the authority of the Project Licence (IACUC permit number: 2016080301). All procedures involving the Huzhu Bamei sows were conducted at the Qinghai Province Huzhu County Bamei Pig Seed Breeding Farm (Huzhu, China) for Scientific Purposes. These Huzhu Bamei sows were experimental animals, and animal feeding followed the recommendations in the ARRIVE guidelines, animals slaughtering followed the American Veterinary Medical Association (AVMA) Guidelines for the Euthanasia of Animals (2020) and was approved by the National Administration of Swine Slaughtering and Quarantine regulations (Qinghai, China).

\subsection{Animals and Diets}

Forty (40) purebred Huzhu Bamei well body condition (score 4) sows were sourced through the Qinghai Province Huzhu County Bamei Pig Seed Breeding Farm (Huzhu, China) having similar body weight (BW), health status, and 3 to 4 years of age being randomly assigned to one of two treatments (20/treatment). The LP treatment diet (12\% CP) was balanced for the five EAA Lys, Met, Thr, Trp, and Val for their standardized ileal digestibility (SID) concentrations and then decreased CP by $2 \%$ compared to a control (CON; $14 \% \mathrm{CP}$ ) diet balanced for the same SID EAA according to Chinese feeding standards for a $90 \mathrm{~kg}$ heavy body conditioned sow. The complete diet composition is given in Table 1 . After $5 \mathrm{~d}$ of facility and diet acclimation, the sows were fed the assigned treatment diet while skipping one estrous cycle (21 days) during natural estrus and then mated. The newborn piglets were maintained with their mothers prior to weaning with litter size, live birth \%, birth weights, and diarrhea rates being published previously (Jin et al., 2019). All sows at all time had ad libitum access to feed and fresh water.

\subsection{Sample Collection}

Randomly, 12 piglets were selected from each treatment group, fasted for 12-hour, weighed, and euthanized with $50 \mathrm{mg} / \mathrm{kg}$ sodium pentobarbital on day 21 of age. The small intestine was ligated at the pylorus, duodenum, jejunum, and ileum and dissected. The ligated jejunum was weighed. The jejunal contents were sampled at approximately the half-way point of the jejunal length, placed into $1.5 \mathrm{~mL}$ sterile polypropylene tubes, and stored in liquid nitrogen until analyses were conducted for intestinal microbiome and metabolome. An approximate $1.5 \mathrm{~cm}$ jejunal tissue sample was collected, washed, and placed in $4 \%$ paraformaldehyde for histomorphometric analysis at the same time.

\subsection{Histomorphometric analysis}


Jejunal tissue samples fixed in $4 \%$ paraformaldehyde were embedded in paraffin $(5 \mu \mathrm{m})$ and stained with HE (hematoxylin-eosin). In each jejunal section, 12 intact villi were randomly selected from each piglet. The jejunum villus height, villus width, crypt depth, and muscular layer thickness were measured using an image analysis system (Caseviewer 2.0 software, 3DHISTECH, Hungary).

\section{5 gDNA Extraction, 16S rRNA Gene Sequencing and Microbial Function Prediction}

The jejunal content samples were extracted to harvest total bacterial DNA using the PowerSoil® DNA Isolation Kit (MO BIO Laboratories, Inc., Carlsbad, CA, USA) according to the manufacturer's instructions. The DNA samples were stored at $-80^{\circ} \mathrm{C}$ until outsourced for analyzing the $16 \mathrm{~s}$ rRNA gene by BIOMARKER (Beijing, China). The 16S rRNA gene sequence (Illumina HiSeq 2500) was used to measure microbial diversity and bacterial community composition. The extracted DNA was used as a template and PCR was performed using barcode primers located on both sides of the V3-V4 hypervariable region of the bacterial 16S rRNA gene. The primer sequences used were: 338F: 5'-ACTCCTACGGGAGGCAGCA-3' and 806R: 5'GGACTACHVGGGTWTCTAAT-3'. Amplification was performed for 30 cycles using a DNA thermal Cycler (Bio-Rad, Hercules, CA, USA). The first cycle was at $98^{\circ} \mathrm{C}$ for 2 min followed by 30 subsequent cycles of $98^{\circ} \mathrm{C} \times 30 \mathrm{~s}, 50^{\circ} \mathrm{C} \times 30 \mathrm{~s}$, then $72^{\circ} \mathrm{C} \times 1 \mathrm{~min}$, and the last cycle at $72^{\circ} \mathrm{C}$ for $7 \mathrm{~min}$.

The original DNA fragments from the raw sequencing reads were merged using FLASH v1.2.7 (http://ccb.jhu.edu/software/FLASH/). The reads were assigned to each sample according to the unique barcodes. The selected high-quality reads were used for bioinformatic analysis. Each sample's unique read was clustered into operational taxonomic units (OTU) based on a $97 \%$ sequence similarity determined accordingly by UCHIME v4.2 (http://drive5.com/usearch/manual/uchime_algo.html).

\subsection{Sample Processing for Metabolomics}

The samples of jejunal contents were thawed at $4^{\circ} \mathrm{C}$. Then, $60 \mathrm{mg}$ were mixed with $200 \mu \mathrm{L}$ ultrapure water to assist in homogenization, followed by adding $800 \mu \mathrm{L}$ of methanol/acetonitrile $(1: 1, \mathrm{v} / \mathrm{v})$. Then samples were vortexed followed by sonication on ice. The samples were incubated for 1 hour at $-20^{\circ} \mathrm{C}$ to remove protein followed by 15 min centrifugation $\left(13,000 \times \mathrm{g}\right.$ at $\left.4^{\circ} \mathrm{C}\right)$, The supernatants were collected followed by vacuum drying followed by storage at $-80^{\circ} \mathrm{C}$ until analyzed using ultra-high-performance liquid chromatography equipped with quadrupole time-of flight mass spectrometry (UPLC-Q-TOF/MS). The quality control (QC) samples were prepared following the same procedures as previously described. For the UPLC-Q-TOF/MS analysis, the samples were re-dissolved in $100 \mu \mathrm{L}$ acetonitrile/water $(1: 1, \mathrm{v} / \mathrm{v})$. Instrument stability and repeatability was monitored using QC samples prepared by pooling $10 \mu \mathrm{L}$ of each sample and analyzed after every 10 experimental samples.

\subsection{UPLC-Q-TOF/MS Analysis}

Jejunal content samples metabolic profiles were measured using an Agilent 1290 Infinity LC system (Agilent Technologies, Santa-Clara, California, USA) coupled with an AB SCIEX Triple TOF 6600 System (AB SCIEX, Framingham, MA, USA). An ACQUITY UPLC BEH Amide $1.7 \mu \mathrm{m}(2.1 \times 100 \mathrm{~mm})$ column for 
both positive and negative models was used for chromatographic separation. The A mobile phase was $25 \mathrm{mM}$ ammonium acetate and $25 \mathrm{mM}$ ammonium hydroxide in water and the B mobile phase was acetonitrile. The solvent gradient was $85 \%$ B mobile phase for 1 min followed by linearly reducing to $65 \%$ by $11 \mathrm{~min}$ followed by further reduction to $40 \%$ in $0.1 \mathrm{~min}$. This mobile phase concentration was maintained for 4 min followed by increasing to $85 \%$ in $0.1 \mathrm{~min}$ increments with a 5 min re-equilibration period.

The ESI source parameters were: Ion Source Gas $1=60$, Ion Source Gas $2=60$, curtain gas $=30$, source temperature $=600^{\circ} \mathrm{C}$, and lonSpray Voltage Floating $\pm 5500 \mathrm{~V}$. In the MS acquisition only mode, the instrument was set to acquire data covering the $\mathrm{m} / \mathrm{z}$ range of 60-1000 Da. The TOF MS scan accumulation time was set at $0.20 \mathrm{~s} / \mathrm{spectra}$ and product ion scan accumulation time was 0.05 $\mathrm{s} /$ spectra. In auto MS/MS acquisition, the instrument was set to acquire data covering the 25-1000 Da $\mathrm{m} / \mathrm{z}$ range, and the product ion scan accumulation time was set at $0.05 \mathrm{~s} / \mathrm{spectra}$. The product ion scan was acquired using information dependent acquisition by selecting the high sensitivity mode. The parameters were set as follows: collision energy fixed at $35 \mathrm{~V}$ with $\pm 15 \mathrm{eV}$; declustering potential at $60 \mathrm{~V}$ $(+)$ and $-60 \mathrm{~V}(-)$; isotopes were excluded within $4 \mathrm{Da}$ and candidate ions were monitored at 10 per cycle.

\subsection{Statistical Analyses}

All data were checked for outliers before any statistical analyses were conducted. Data were either plotted or the box and whisker plots and the Shapiro Wilk Test were used to verify that the data were normally distributed $(P>0.15)$. All data were subjected to least squares analysis of variance (ANOVA) for a completely random design (CRD; Steel and Torrie, 1980) having 2 treatments using SPSS 21 software (SPSS Inc., Chicago, IL, USA). The statistical linear additive model was:

$Y_{i}=\mu+T+e_{i}$

Where $Y_{i}=$ depended variable, $\mu$ - overall mean, $T=$ treatment of $L P$ or CON and $e_{i}=$ residual random error. Least squares means were separated using the Least Significant Difference (LSD) and significant was declared at $\mathrm{P}<0.05$.

Microbial Data Analysis. The OTU were rarified based on several metrics for alpha diversity analysis including OTU rank curves, rarefaction, and Shannon, along with Shannon, Chao1, Simpson, and ACE calculated indices. Principal Coordinates Analysis (PCOA) and unweighted pair group method with arithmetic mean (UPGMA) were performed using QIIME based weighted unifrac distance for beta diversity analysis (Jin et al., 2019). Finally, PICRUSt (Parks et al., 2014) was used to predict microbial function. Bacterial domains, phyla, and genera were compared using Wilcoxon rank-sum test, with the FDR adjusted $P$ value $<0.05$ being considered as significantly different. Finally, Spearman's rank correlations among jejunal microbiome changes, histomorphometric, and shifted metabolome were calculated to examine functional impacts of material LP diet concentrations on the small intestinal microbiome. 
Metabolomics Data Analysis. UPLC-Q-TOF/MS raw data were converted to mzXML files using Proteo Wizard MSconventer tool and then processed using XCMS online software (https://bioconductor.org/packages/release/bioc/html/xcms.html). The XCMS parameters were: feature detection centwave settings $(\Delta \mathrm{m} / \mathrm{z}=25 \mathrm{ppm}$, peakwidth $=c(10,60))$; retention time correction obiwarp settings (profStep $=1$ ); and minfrac parameters $=0.5$, bw $=5$ and mzwid $=0.025$ for chromatogram alignment. After being normalized and integrated using support vector regression, the processed data were uploaded into MetaboAnalyst 4.0 software for further evaluation (www.metaboanalyst.ca). Orthogonal partial least square discriminant analysis (OPLS-DA) and 3D-Principal Component Analysis (3D-PCA) for both positive and negative models were performed after log transformation and pareto scaling. For each variable, the variable importance projection (VIP) value in the OPLS-DA model was calculated to determine the classification contribution. Metabolites having VIP values $>1$ were further evaluated using Student's t-test at univariate level for each metabolite with $\mathrm{P}<0.05$ considered as statistically significant. Changes in microbial community metabolite profile can reflect microbial community dynamic alterations. Therefore, defining relationships between metabolic function and microbial community structure via microbial and metabolomics data using correlation analysis may provide insight for a comprehensive understanding of microbial composition and community function.

\section{Results}

\subsection{Piglet Performance}

Piglet birth BW (day 0) was greater for sows fed LP compared with piglet birth BW for sows fed CON, while $21 \mathrm{~d}$ piglet BW tended $(P<0.07)$ to be greater for piglets from sows fed LP compared with sows fed CON (Table 2). However, these initial and final piglet BW differences did not affect piglet ADG, which was similar $(P>0.36)$ among both treatments.

\subsection{Jejunal Morphology}

Intestinal HE staining demonstrated that piglets nursing sows fed a maternal LP diet demonstrated reduced $(P<0.05)$ villus height and ratio of villus height to crypt depth, while jejunum relative weight, villus width, crypt depth, and muscle thickness were increased $(P<0.05)$ compared with piglets from sows fed the maternal CON diet (Table 3).

\subsection{The Diversity and Composition of Jejunal Microbiota}

The 16S RNA jejunal microbiota samples after data filtering, quality control, and low-confidence singletons removal resulted in an average of $42,718 \mathrm{~V}_{3}-\mathrm{V}_{4} 16 \mathrm{~S}$ rRNA gene sequence reads being obtained for the $21 \mathrm{~d}$ samples (two piglet litters were not yet weaned due to late farrowing). The sequence lengths ranged from 415 up to $429 \mathrm{bp}$. The rarefaction curves resulted in new OTU diminishing identification rates with increasing number of reads per sample. This implies that the jejunum bacterial community has adequate sampling depth for identifying dominant members. Similarly, the Good's coverages exceeded $99 \%$ demonstrating excellent sequence accuracy and reproducibility (Table 4). Of the 482 total OTU numbers, 452 OTU were detected in both groups. Based on the Shannon $(P<0.001)$, and Simpson $(P=$ 
0.001) indices piglets from the maternal fed LP diet demonstrated more diversity and greater evenness compared with piglets from the material fed CON diet (Table 4). The Chaol $(P=0.519)$ and Ace $(P=$ 0.435 ) indices were similar for piglets from the maternal fed LP compared with the maternal fed CON. Taxonomic analysis revealed the predominant phyla Firmicutes and Proteobacteria being $67.21 \%$ and $24.97 \%$, respectively of total reads identifying 16 bacterial phyla. (Figure 1A). At the genus level, 232 genera were identified in the jejunal samples. The predominant genera were Lactobacillus $(51.11 \%)$, Escherichia-Shigella (9.00\%), Actinobacillus (7.41\%), Clostridium_sensu_stricto_1 (5.60\%), Romboutsia (4.35\%), and Buchnera (3.54\%), respectively (Figure 1B).

Furthermore, using a PCoA plot illustrated microbial community dissimilarity and revealed distinct structures between piglets from the maternal fed LP compared with maternal fed CON (Figure 1C). The PCoA plot uses a weighted method for unifrac similarity, which revealed PC1 and PC2 explained 55.61\% and $13.98 \%$ of sample variation, respectively. Similarly, the jackknifed beta diversity and hierarchical clustering analysis via the Unweighted Pair-group Method with Arithmetic Mean (UPGMA) demonstrated that different piglets fed different maternal CP diets were clustered in their individual groups (Figure 1D). In addition, piglets from maternal fed CON diets in the PCoA plot were clustered into two subgroups (Figure 1C) and UPGMA hierarchical clustering analysis (Figure 1D), which was attributed to individual variations of jejunum microbiome profiles.

\subsection{Differences in Jejunal Bacterial Community Composition}

Relative phylum abundances of Firmicutes, Proteobacteria, Bacteroidetes, and unknown were $>1 \%$ for both treatments (Table 5). Firmicutes relative abundance was decreased $(P=0.002)$ and Proteobacteria $(P=0.001)$ was increased for piglets from the maternal LP treatment compared with piglets from the sows fed maternal CON. Thirty-two (32) specific genera demonstrated relative abundances $>0.1 \%$. The relative bacterial community abundances of Escherichia-Shigella $(P=0.050)$, Actinobacillus $(P=0.050)$, Clostridium_sensu_stricto_1 $(P=0.003)$, Veillonella $(P=0.015)$, and Turicibacter $(P=0.011)$ were higher and Lactobacillus was lower $(P<0.001)$ for piglets from the maternal fed LP treatment compared with piglts from the maternal fed CON treatment (genus level; Table 6).

The receiver operating characteristic curve (ROC) predicted different microorganisms for piglets from maternal fed LP compared to maternal fed CON piglets for inducing jejunal development. The area under the curve (AUC) judged via diagnosis test (Xia et al., 2013) that Lactobacillus is the most likely biomarker $(0.9<$ AUC < 1.0) for piglets from both treatments, while Clostridium_sensu_stricto_1 and Turicibacter are more likely biomarkers $(0.8<$ AUC $<0.9)$ for piglets from maternal fed LP sows.

\subsection{Predicted Function of Jejunal Microbiota}

The PICRUSt analyzed pathway compositions for evaluating jejunal bacterial community functional capacity is a functional-gene-count matrix. Second level KEGG (levels) metabolism pathway analysis via

global and overview maps demonstrated that biosynthesis of other secondary metabolites were enriching amino acid, cofactors, and vitamins metabolism $(P<0.05)$, while lipid and nucleotide metabolism were 
decreased $(P<0.05)$ for piglets when maternal sows were fed LP diet compared with piglets from the maternal fed CON (Figure 2).

\subsection{Correlations between Intestinal Microbial Species and Jejunum Morphological Traits}

Numerous correlations via Spearman's correlation analyses (correlation coefficient $\mid>$ or $<0.4, P<0.05$, Figure 3) were investigated between the different genera $(n=6)$ relative abundances and morphological parameters $(n=7)$. Clostridium_sensu_stricto_1 was positively correlated with villus width, crypt depth, and muscular thickness, while being negatively correlated with villus height, and ratio of villus height: crypt depth. Escherichia-Shigella was positively correlated with muscular thickness and negatively correlated with villus height. Turicibacter was positively correlated with crypt depth and muscular thickness, while Veillonella was positively correlated with villus width. Lactobacillus was positively correlated with villus height, and villus height: crypt depth, and negatively correlated with jejunum weight, villus width, crypt depth, and muscular thickness.

\subsection{Jejunum Metabolites and Metabolic Pathways}

The 3D-PCA and OPLS-DA multivariate statistical analysis models were applied to evaluate the different group classifications via Score plots (Figure 4). The 3D-PCA Score plots were derived from the LCTOF/MS jejunal metabolic profiles demonstrated separation between the LP and CON fed diets. A clear separation and discrimination were observed between the two groups, which indicated that the OPLS-DA model could be used to identify piglet differences between maternal fed LP and CON diets. In addition, the volcano plots highlight the 44 metabolites being altered $(V I P>1.0$ and $P<0.05)$ for piglets from the maternal fed LP fed treatment (Table 7). Thirty-four (34) metabolites were increased and 10 metabolites were decreased in piglets from the maternal fed LP diet compared with piglets from the maternal fed CON (Table 7). These amino acids, nucleotides, lipids, organic acids, and numerous metabolites are involved in multiple jejunum biochemical processes of the Bamei piglet. The hierarchical clustering analysis (HCA) with a heat map was performed to visualize the Bamei piglet jejunum metabolome differences associated with two maternal CP concentrations. The positive ionization data (Figure 5) and negative ionization data (Figure 6) clearly demonstrate similar clustering patterns of molecular features within each treatment. The maternal CP concentration demonstrated an impact $(P<0.05)$ on jejunum metabolome, while cluster differences were clearly observable in the HCA generated heatmap plot. The AUC value for each metabolite was calculated, such that metabolites with an AUC > or equal to 0.85 were selected as potential signatures. Six metabolites determine via the AUC elimination step (Figure 7A) were: L-Methionine (AUC = 0.875), Taurochenodeoxycholate (AUC = 0.882), Taurodeoxycholic acid (AUC = 0.875), Tauroursodeoxycholic acid $(A U C=0.896)$, 4-Androsten- 17 beta-ol-3-one glucosiduronate $(A U C=$ 0.931), and Cholic acid (AUC $=0.882)$. Furthermore, the metabolic pathway enrichment analysis (Figure 7B) demonstrated that feeding a maternal LP diet altered $(P<0.05$; rich factor $>0.10)$ Arg, Cys, His, Met, Phe, Try, Tyr, and linoleic acid metabolism, biosynthesis of Phe, Tyr, and Try, and Lys degradation. Consistent with the PICRUSt function prediction pathway, amino acid metabolism was enriched for 
piglets from sows fed a maternal LP concentration diet compared with piglets from sows fed a maternal CON.

\subsection{Correlations between Differential Genera and Metabolites}

The functional correlation between intestinal microbiome changes and metabolite perturbations (VIP $>2$, $\mathrm{P}<0.05)$ was evaluated using a correlation matrix generated by calculating the Spearman's correlation coefficient. Clear identifiable correlations between perturbed intestinal microbiome and altered metabolite profiles were found $(r>0.5$ or $<-0.5, P<0.05)$. Clostridium_sensu_stricto_ 1 was negatively correlated with L-His; Lactobacillus was positively correlated with chenodeoxycholate, cholic acid, glycocholic acid, L-His, L-Leu, L-Met, L-Try, L-Val, taurochenodeoxycholate, taurodeoxycholic acid, and tauroursodeoxycholic acid, but was negatively correlated with hypoxanthine, linoleic acid, palmitoyl ethanolamide, PC (16:0/16:0), and uracil; Turicibacter was negatively correlated with the D-Pro; Veillonella was positively correlated with uracil (Figure 8). In summary, dietary maternal CP concentrations induced a piglet intestinal microbiome taxonomic perturbation, which in turn substantially alters the intestinal metabolomic profile as observed due to changes in the diverse intestinal microbiota-related metabolites.

\section{Discussion}

In this study, reducing maternal dietary protein concentrations by $2 \%$ units resulted in similar $21 \mathrm{~d}$ ADG. The main nutrient digestion and absorption site is the jejunum (Low, 1976). Maternal suckled milk enters the piglet's gastrointestinal tract, thereby promoting crypt cell proliferation and proliferation. Suckling piglet jejunal development directly affects post-weaning growth performance (Buddington and Sangild, 2011). The small intestinal growth rate before and after birth of the piglet is greater than the whole body (Cheng and Leblond, 1974). The small intestine relative weight $24 \mathrm{~h}$ after birth is $50 \%$ greater than at birth (Adeola and King, 2006). Intestinal crypt depth increases $40 \%$ and villus height increases $35 \%$ within $3 \mathrm{~d}$ (Godlewski et al., 2005). These crypt stem cells divide and differentiate to form intestinal epithelial cells that gradually migrate to the villi tip (Godlewski et al., 2005) for nutrient absorption.

The small intestine has an important role in defense against health challenges in addition to nutrient digestion and absorption. These new intestinal epithelial cells replace intestinal villi epithelial cells loss caused by apoptosis, while allowing the piglet to adapt to intestinal adversity. Through this process, the digestive and absorption functions of intestinal epithelial cells are gradually improved (Wang et al., 2016; Papadopoulos et al., 2017). To some extent, the crypt depth of small intestinal villi reflects the production efficiency of intestinal epithelial cells. The villus height / crypt depth reflects the overall physiological function of the small intestine. AS muscle thickness increases, the intestinal villi muscle fibers and rhythmic contractions increase, which improves mechanical digestion efficiency and nutrient absorption.

The combined data using Bamei piglets demonstrated that maternal dietary LP concentrations resulted in significant changes in intestinal microbiome composition compared with CON piglets. The altered intestinal microbial compositions were strongly associated with numerous changes of intestinal microbiota-related metabolites. These data demonstrate that a $2 \%$ reduction in dietary $\mathrm{CP}$ with similar 
SID AA concentrations not only alters intestinal bacteria abundance levels, but alters intestinal microbiome metabolic profile, which makes the homeostasis of host metabolites rebuilt. These findings may provide useful insights into a mechanism of feeding a maternal diet for altering the piglet's intestinal microbiome as an alternative mechanism of using dietary intervention for disease treatment.

After the piglet's birth, there are 2 sources of gut microbes with one being the maternal microbes, which are vertically passed, while the 2 nd source is environmental, which are horizontally passed. In agreement with previous pig studies (Fan et al., 2017; Yan et al., 2017; Chen et al., 2018; He et al., 2019; Wang et al., 2019), the Bamei piglet's dominant jejunum core microbiome was the phyla Firmicutes, Proteobacteria, and Bacteroidetes. The dominant genus level Bamei suckling piglet jejunum bacteria were: Lactobacillus, Escherichia-Shigella, Actinobacillus, Buchnera, Romboutsia, and Clostridium_sensu_stricto_1. The bacterial community diversity and richness are known to be influenced by dietary intervention (Smits et al., 2016; Johnson et al., 2019a). Alpha diversity metrics (Shannon and Simpson index) demonstrated a higher piglet bacterial diversity from sows fed lower maternal dietary CP concentrations compared with piglets from sows fed the CON CP concentrations, suggesting that altering CP concentration has a direct impact on jejunal microbial composition of Bamei suckling piglets.

Lactobacillus are beneficial bacterial members of the small intestinal microbiota that were reduced for piglets from sows fed the LP diet. The intestinal bacterial environment can protect the intestine from toxic dietary ingredients (Di Rienzi et al., 2018). The reduction of Lactobacillus spp. abundance may result from decreased oligosaccharide ingestion (less soybean meal inclusion), which reduces nutrient availability, which relates to reduced piglet weight (Drissi et al., 2017). These results indicate that maternal dietary LP concentration alters Bamei piglets intestinal microbiota through altering the beneficial bacterial colony structure (Bian et al., 2016). Therefore, it is reasonable to hypothesize that intestinal microbiota differences are the result of early dietary intervention, host-microbe interactions, and/or host physiological state. The most important host-microbe interaction may occur on or at the intestinal barrier.

The metabolomics data revealed that maternal dietary CP concentration alters jejunal metabolite concentration indicating that jejunal metabolism may be linked with jejunal microbiota activity. Maternal LP concentration altered numerous metabolite concentrations associated with piglet protein digestion and absorption, as well as, amino acid biosynthesis. Amino acids are key precursors for protein and polypeptide synthesis and regulators of some metabolic pathways mainly derived from jejunal microbiota and host enzymatic degradation of dietary proteins and microproteins. The piglet L-Met concentration from sows fed the LP diet was higher compared with piglets from sows fed the CON diet. LMet is a precursor to other sulfur-containing AA, including homocysteine, which are important for protein synthesis, polyamine formation, and synthesis of many metabolites. Additionally, L-Met plays important roles in intestinal bacteria cell protein synthesis and regulating mucosal antigen responses (Mariz et al., 2018). Bamei piglet jejunal linoleic acid metabolism was altered by dietary CP concentrations, which speculating could be contributing to improving intestinal barrier function by enhancing the GPR40-MEKERK pathway contributing to improved intestinal barrier integrity (Miyamoto et al., 2015; Di Rienzi et al., 
2018). Further studies are required to identify the AA source from either microbiota or host metabolism (diet and endogenous), while examining the effects on in vivo intestinal function and immune development.

This study demonstrates that dietary intervention can alter intestinal microbiota composition, function, diversity and activity to reduce protein supplementation and feed cost for maintaining host health by altering metabolite availability (such as amino acids; Li et al., 2018; Johnson et al., 2019b; KovatchevaDatchary et al., 2019). The Spearman's correlation analysis suggested changes in jejunal microbial abundance induced by feeding lower maternal dietary protein concentrations resulted in a shift in the microbial metabolome.

The correlation analysis between intestinal bacteria (Clostridium_sensu_stricto_1, Lactobacillus, and Turicibacter) and metabolites demonstrated that feeding a maternal LP diet can induce shifting abundance changes in the piglet's intestinal microbiome. Equally important, dietary interventions may not always alter the piglet's bacterial species and abundance but may alter the metabolites produced by these bacterial species thru influencing their metabolism and physiology. Therefore, changes in the metabolic profile of the gut microbiome may not completely depend on altering the microbial profile that was revealed by $16 \mathrm{~S}$ rRNA gene sequencing. In addition to influencing bacterial types, intestinal numbers, metabolism and physiology, this metabolic change might be achievable through other mechanisms, such as regulating bacteria gene and protein expression. Lin et al. (Lin et al., 2019) demonstrated that acetate and butyrate production by the intestinal microbiome mediated the regulation of growth-related genes in the rumen epithelium which regulated many epithelial physiological processes. So, future research should focus on multi-omics methods to provide more information sources for the intestinal health of piglets when feeding maternal LP diets.

These data demonstrated that dietary $\mathrm{CP}$ concentrations altered the intestinal microbiome composition and associated metabolic profiles in Bamei piglets. This is the first key step to understand the impacts of feeding low protein diets influencing intestinal microbiome and function. This could be an exciting research field with the potential to solve many important problems. For example, does altering maternal dietary protein concentration influence intestinal microbiota and related metabolites for newborn piglets to improve growth performance and feed efficiency remains to be determined. The maternal intestinal microbiota largely determines infant intestinal microbiota, which Maqsood et al. (2019) demonstrated that $63 \%$ of the infant's bacterial flora originated via the maternal microbiota. Although dietary protein was reduced by $2 \%$ units for lactating Bamei sows, their dietary protein requirements during the gestation period are lower (NRC, 2012). Therefore, it may be necessary to determine CP concentration available for the intestinal microbiome at different pregnancy stages having similar SID EAA concentrations. What may be critically important is the impact of protein on piglet intestinal microbiome, due to bacterial flora during pregnancy affecting fetal development (McDonald and McCoy, 2019), and it's impact on the bacterial flora development of infants (Baumann-Dudenhoeffer et al., 2018; Parnanen et al., 2018).

\section{Conclusions}


These data clearly demonstrates that maternal diet CP concentration alters the piglet's intestinal microbiome composition for altered metabolite concentrations used in various metabolic pathways when diet $\mathrm{CP}$ was reduced $2 \%$ units with similar EAA concentrations. Reducing maternal dietary CP demonstrated altered piglet histomorphology, microbiota composition and function, while modulating jejunum microbiota metabolic profiles that are associated with specific intestinal bacteria genera. These alterations aid in understanding the beneficial impacts of feeding maternal LP diets without affecting SID EAA concentrations on piglet intestinal health. Furthermore, the regulated intestinal microbiome-related metabolites may be potential biomarkers to be used in the future to explore functional impacts of maternal dietary interventions on the piglet's intestinal microbiome.

\section{Declarations}

\section{Acknowledgments}

The authors are grateful to all the participants who took part in this study.

This research was funded by the Key of laboratory of Plateau Ecology and Agriculture, Qinghai University (2019-zz-02) and National Natural Science Foundation of China (31660662).

\section{References}

1. Adeola O, and King D E. 2006. Developmental changes in morphometry of the small intestine and jejunal sucrase activity during the first nine weeks of postnatal growth in pigs. Journal of Animal Science 84, 112-118.

2. Baumann-Dudenhoeffer A M, D'souza A W, Tarr P I, Warner B B, and Dantas G. 2018. Infant diet and maternal gestational weight gain predict early metabolic maturation of gut microbiomes. Nat Med 24, 1822-1829.

3. Bian G, Ma S, Zhu Z, Su Y, Zoetendal E G, Mackie R, Liu J, Mu C, Huang R, and Smidt H. 2016. Age, introduction of solid feed and weaning are more important determinants of gut bacterial succession in piglets than breed and nursing mother as revealed by a reciprocal cross-fostering model. Environmental Microbiology 18, 1566-1577.

4. Buddington, R. K., and P. T. Sangild. 2011. Companion animals symposium: Development of the mammalian gastrointestinal tract, the resident microbiota, and the role of diet in early life. J. Anim. Sci. 89:1506-1519. https://doi.org10.2527/jas.2010-3705

5. Chen X, Song P, Fan P, He T, Jacobs D, Levesque C L, Johnston L J, Ji L, Ning M, and Chen Y. 2018. Moderate Dietary Protein Restriction Optimized Gut Microbiota and Mucosal Barrier in Growing Pig Model. Frontiers in Cellular \& Infection Microbiology 8, 246.

6. Cheng H, and Leblond C P. 1974. Origin, differentiation and renewal of the four main epithelial cell types in the mouse small intestine. V. Unitarian Theory of the origin of the four epithelial cell types. American Journal of Anatomy 141, 537-561. 
7. Dai Z L, Zhang J, Wu G, and Zhu W Y. 2010. Utilization of amino acids by bacteria from the pig small intestine. Amino Acids 39, 1201-1215.

8. Di Rienzi S C, Jacobson J, Kennedy E A, Bell M E, Shi Q, Waters J L, Lawrence P, Brenna J T, Britton R A, Walter J, and Ley R E. 2018. Resilience of small intestinal beneficial bacteria to the toxicity of soybean oil fatty acids. Elife 7, e32581.

9. Drissi F, Raoult D, and Merhej V. 2017. Metabolic role of lactobacilli in weight modification in humans and animals. Microb Pathog 106, 182-194.

10. Fan P, Liu P, Song P, Chen X, and Ma X. 2017. Moderate dietary protein restriction alters the composition of gut microbiota and improves ileal barrier function in adult pig model. Sci Rep 7 , 43412.

11. Godlewski M M, Slupecka M, Wolinski J, Skrzypek T, Skrzypek H, Motyl T, and Zabielski R. 2005. Into the unknown--the death pathways in the neonatal gut epithelium. Journal of Physiology and Pharmacology 56 Suppl 3, 7-24.

12. He, Y., J. Deen, G. C. Shurson, L. Wang, C. Chen, D. H. Keisler, and Y. Z. Li. 2016. Identifying factors contributing to slow growth in pigs. J. Anim. Sci. 64:2103-2116.

13. He M, Gao J, Wu J, Zhou Y, Fu H, Ke S, Yang H, Chen C, and Huang L. 2019. Host Gender and Androgen Levels Regulate Gut Bacterial Taxa in Pigs Leading to Sex-Biased Serum Metabolite Profiles. Frontiers in Microbiology 10, 1359.

14. Holmes A J, Chew Y V, Colakoglu F, Cliff J B, Klaassens E, Read M N, Solon-Biet S M, Mcmahon A C, Cogger V C, Ruohonen K, Raubenheimer D, Le Couteur D G, and Simpson S J. 2017. Diet-Microbiome Interactions in Health Are Controlled by Intestinal Nitrogen Source Constraints. Cell Metab 25, 140151.

15. Huttenhower C, Gevers D, Knight R, Abubucker S, Badger J H, Chinwalla A T, Creasy H H, Earl A M, Fitzgerald M G, and Fulton R S. 2012. Structure, function and diversity of the healthy human microbiome. Nature 486, 207-214.

16. Jain A, Li X H, and Chen W N. 2019. An untargeted fecal and urine metabolomics analysis of the interplay between the gut microbiome, diet and human metabolism in Indian and Chinese adults. Scientific Reports 9, 9191.

17. Jin, Y. C. 2006. The protection and ultuilization of Qinghai Bamei prig. Swine Ind. Sci. 23:70-74.

18. Jin J, Zhang L, Jia J, Chen Q, Yuan Z, Zhang X, Sun W, Ma C, Xu F, Zhan S, Ma L, and Zhou G. 2019. Effects of Maternal Low-Protein Diet on Microbiota Structure and Function in the Jejunum of Huzhu Bamei Suckling Piglets. Animals 9, 713.

19. Johnson A J, Vangay P, Al-Ghalith G A, Hillmann B M, Ward T L, Shields-Cutler R R, Kim A D, Shmagel A K, Syed A N, Walter J, Menon R, Koecher K, and Knights D. 2019a. Daily Sampling Reveals Personalized Diet-Microbiome Associations in Humans. Cell Host Microbe 25, 789-802.e785.

20. Johnson A J, Vangay P, Al-Ghalith G A, Hillmann B M, Ward T L, Shields-Cutler R R, Kim A D, Shmagel A K, Syed A N, Walter J, Menon R, Koecher K, and Knights D. 2019b. Daily Sampling Reveals Personalized Diet-Microbiome Associations in Humans. Cell Host Microbe 25, 789-802.e785. 
21. Kovatcheva-Datchary P, Shoaie S, Lee S, Wahlstrom A, Nookaew I, Hallen A, Perkins R, Nielsen J, and Backhed F. 2019. Simplified Intestinal Microbiota to Study Microbe-Diet-Host Interactions in a Mouse Model. Cell Reports 26, 3772-3783.e3776.

22. Kreznar J H, Keller M P, Traeger L L, Rabaglia M E, Schueler K L, Stapleton D S, Zhao W, Vivas E I, Yandell B S, and Broman A T. 2017. Host Genotype and Gut Microbiome Modulate Insulin Secretion and Diet-Induced Metabolic Phenotypes. Cell Reports 18, 1739-1750.

23. Kuang Z, Wang Y, Li Y, Ye C, Ruhn K A, Behrendt C L, Olson E N, and Hooper L V. 2019. The intestinal microbiota programs diurnal rhythms in host metabolism through histone deacetylase 3 . Science $365,1428-1434$.

24. Kuntz T M, and Gilbert J A. 2017. Introducing the Microbiome into Precision Medicine. Trends Pharmacol Sci 38, 81-91.

25. Li Y, Fu X, Ma X, Geng S, Jiang X, Huang Q, Hu C, and Han X. 2018. Intestinal MicrobiomeMetabolome Responses to Essential Oils in Piglets. Frontiers in Microbiology 9, 1988.

26. Lin L, Xie F, Sun D, Liu J, Zhu W, and Mao S. 2019. Ruminal microbiome-host crosstalk stimulates the development of the ruminal epithelium in a lamb model. Microbiome 7, 83.

27. Low, A. G. 1976. Digestion and absorption of nutrients in growing pigs. Proc. Nutr. Soc. 35:57-62. http://doi.org/10.1079/PNS19760009

28. Maqsood R, Rodgers R, Rodriguez C, Handley S A, Ndao I M, Tarr P I, Warner B B, Lim E S, and Holtz L R. 2019. Discordant transmission of bacteria and viruses from mothers to babies at birth. Microbiome 7, 156-169.

29. Mariz L D S, Amaral P M, Valadares Filho S C, Santos S A, Detmann E, Marcondes M I, Pereira J M V, Silva Junior J M, Prados L F, and Faciola A P. 2018. Dietary protein reduction on microbial protein, amino acid digestibility, and body retention in beef cattle: 2 . Amino acid intestinal absorption and their efficiency for whole-body deposition. Journal of Animal Science 96, 670-683.

30. Martinez-Lopez M, Iborra S, Conde-Garrosa R, Mastrangelo A, Danne C, Mann E R, Reid D M, Gaboriau-Routhiau V, Chaparro M, Lorenzo M P, Minnerup L, Saz-Leal P, Slack E, Kemp B, Gisbert J P, Dzionek A, Robinson M J, Ruperez F J, Cerf-Bensussan N, Brown G D, Bernardo D, LeibundgutLandmann S, and Sancho D. 2019. Microbiota Sensing by Mincle-Syk Axis in Dendritic Cells Regulates Interleukin-17 and -22 Production and Promotes Intestinal Barrier Integrity. Immunity 50, 446-461.e449.

31. Mcdonald B, and Mccoy K D. 2019. Maternal microbiota in pregnancy and early life. Science 365, 984-985.

32. Miyamoto J, Mizukure T, Park S B, Kishino S, Kimura I, Hirano K, Bergamo P, Rossi M, Suzuki T, Arita M, Ogawa J, and Tanabe S. 2015. A gut microbial metabolite of linoleic acid, 10-hydroxy-cis-12octadecenoic acid, ameliorates intestinal epithelial barrier impairment partially via GPR40-MEK-ERK pathway. J Biol Chem 290, 2902-2918.

33. National Research Council. 2012. Nutrient requirements of swine. 11th revised ed. National Academy of Sciences. Washington, DC. 
34. Papadopoulos G A, Poutahidis T, Tallarico N, Hardas A, Teliousis K, Arsenos G, and Fortomaris P D. 2017. Dietary supplementation of encapsulated organic acids enhances performance and modulates immune regulation and morphology of jejunal mucosa in piglets. Res Vet Sci 115, 174-182.

35. Parks D H, Tyson G W, Philip H, and Beiko R G. 2014. STAMP: statistical analysis of taxonomic and functional profiles. Bioinformatics 30, 3123-3124.

36. Parnanen K, Karkman A, Hultman J, Lyra C, Bengtsson-Palme J, Larsson D G J, Rautava S, Isolauri E, Salminen S, Kumar H, Satokari R, and Virta M. 2018. Maternal gut and breast milk microbiota affect infant gut antibiotic resistome and mobile genetic elements. Nature communication 9, 3891.

37. Pattaroni C, Watzenboeck M L, Schneidegger S, Kieser S, Wong N C, Bernasconi E, Pernot J, Mercier L, Knapp S, Nicod L P, Marsland C P, Roth-Kleiner M, and Marsland B J. 2018. Early-Life Formation of the Microbial and Immunological Environment of the Human Airways. Cell Host Microbe 24, 857865.e854.

38. Peisl B Y L, Schymanski E L, and Wilmes P. 2018. Dark matter in host-microbiome metabolomics: Tackling the unknowns-A review. Anal Chim Acta 1037, 13-27.

39. Rist V T, Weiss E, Eklund M, and Mosenthin R. 2013. Impact of dietary protein on microbiota composition and activity in the gastrointestinal tract of piglets in relation to gut health: a review. Animal 7, 1067-1078.

40. Smits S A, Marcobal A, Higginbottom S, Sonnenburg J L, and Kashyap P C. 2016. Individualized Responses of Gut Microbiota to Dietary Intervention Modeled in Humanized Mice. mSystems 1 , e00098-00016.

41. Steel, R. G. D., and J. H. Torrie. 1980. Principles and procedures of statistics. 2nd ed. McGraw-Hill Book Co., New York, NY.

42. Wang J, Zeng L, Tan B, Li G, Huang B, Xiong X, Li F, Kong X, Liu G, and Yin Y. 2016. Developmental changes in intercellular junctions and $\mathrm{Kv}$ channels in the intestine of piglets during the suckling and post-weaning periods. Journal of Animal Science \& Biotechnology 7, 228-237.

43. Wang X, Tsai T, Deng F, Wei X, Chai J, Knapp J, Apple J, Maxwell C V, Lee J A, Li Y, and Zhao J. 2019. Longitudinal investigation of the swine gut microbiome from birth to market reveals stage and growth performance associated bacteria. Microbiome 7, 109.

44. Wang Y, Zhou J, Wang G, Cai S, Zeng X, and Qiao S. 2018. Advances in low-protein diets for swine. Journal of Animal Science \& Biotechnology 9, 23-36.

45. Xia J, Broadhurst D I, Wilson M, and Wishart D S. 2013. Translational biomarker discovery in clinical metabolomics: an introductory tutorial. Metabolomics 9, 280-299.

46. Yan, S, Zhu C, Yu T, Huang W, Huang J, Kong Q, Shi J, Chen Z, Liu Q, Wang S, Jiang Z, and Chen Z. 2017. Studying the Differences of Bacterial Metabolome and Microbiome in the Colon between Landrace and Meihua Piglets. Frontiers in Microbiology 8, 1812.

47. Yang, B. C., and S. B. Gun. 2007. Current situation and in vivo protection method of Qinghai Bamei Pig. Chin. Qighai J. Anim. Vet. Sci. 37:31-32. 
48. Yang, G. S., and Y. Song, 1991. Study on the chemical composition of the meat of Bamei pig meat. J. Northwest Univ. 4:38-42.

49. Zhang Q, and Reed M. 2008. Examining the Impact of the World Crude Oil Price on China's Agricultural Commodity Prices: The Case of Corn, Soybean, and Pork The Southern Agricultural Economics Association Annual Meeting, Dallas, TX.

\section{Tables}

\section{Table 1}

Ingredient and nutrient composition of maternal diets (DM basis) containing $12 \%$ (LP) or $14 \%$ crude protein $(\mathrm{CON})$. 


\begin{tabular}{lll} 
Items & \multicolumn{2}{l}{$\%$ of DM } \\
\cline { 2 - 3 } & LP & CON \\
\hline Ingredient composition & & \\
\hline Corn & 50.60 & 44.90 \\
\hline Soybean meal & 4.50 & 9.80 \\
\hline Rapeseed meal & 2.50 & 2.70 \\
\hline Wheat bran & 37.78 & 38.14 \\
\hline Lys & 0.34 & 0.20 \\
\hline Met & 0.07 & 0.05 \\
\hline Thr & 0.15 & 0.10 \\
\hline Trp & 0.02 & 0.01 \\
\hline Val & 0.04 & 0.10 \\
\hline $4 \%$ premixb & 4.00 & 4.00 \\
\hline Nutrient concentrations, calculated via formulation. \\
\hline DE (MJ/kg) a & 11.72 & 11.72 \\
\hline CPb & 12.04 & 12.04 \\
\hline Lys & 0.81 & 0.81 \\
\hline Met+Cys & 0.33 & 0.33 \\
\hline Thr & 0.35 & 0.35 \\
\hline Trp & 0.08 & 0.08 \\
\hline Val & 0.26 & 0.26 \\
\hline Total Ca & 0.62 & 0.62 \\
\hline Total P & 0.51 & 0.51 \\
\hline Salt & 3.20 & 3.20 \\
\hline
\end{tabular}

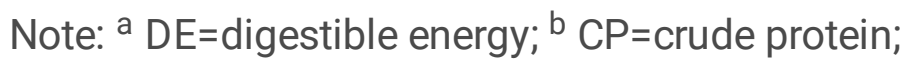

b The premix during pregnancy provided the following per kilogram of diets: vitamin A: $3.52 \mathrm{kIU}$; vitamin E: 20 kIU; vitamin D3: 0.76 kIU; vitamin K3: 2.6 mg; vitamin B2: 9.52 mg; vitamin B3: 24 mg; vitamin B5: 45 mg; Cu: 4 mg; Fe: 10 mg; Zn: 40 mg; Mn: 16 mg; Ca: 15 \%; Total P: 1.8 \%; NaCl: 8 \%; Water: $10 \%$. 
Table 2

Piglet body weight (BW) and average daily gain (ADG) when feeding maternal diets containing $12 \%$ (LP) or $14 \%$ crude protein (CON).

\begin{tabular}{|lllll|}
\hline Items & LP & CON & SDM & P-value \\
\hline Piglet BW, kg & & & & \\
\hline Day 0 & 0.90 & 0.88 & 0.02 & 0.020 \\
\hline Day 21 & 3.85 & 3.78 & 0.09 & 0.067 \\
\hline ADG, $0-21, \mathrm{~g} / \mathrm{d}$ & 135.8 & 134.0 & 1.38 & $<0.37$ \\
\hline
\end{tabular}

Table 3

Jejunum weight and tissue morphology by 21-day old suckling piglets when feeding maternal diets containing $12 \%$ (LP) or $14 \%$ crude protein (CON).

\begin{tabular}{|lllll|}
\hline Items & LP & CON & SDM & P-value \\
\hline Jejunum weight, g & 123.22 & 109.95 & 17.12 & 0.074 \\
\hline Jejunum relative weight, \% & 3.42 & 3.17 & 0.30 & 0.048 \\
\hline Villus height, $\mu \mathrm{m}$ & 318.58 & 385.44 & 17.99 & $<0.001$ \\
\hline Villus width, $\mu \mathrm{m}$ & 96.44 & 83.43 & 3.62 & $<0.001$ \\
\hline Crypt depth, $\mu \mathrm{m}$ & 150.15 & 99.01 & 6.58 & $<0.001$ \\
\hline Villus height: Crypt depth & 2.13 & 4.62 & 0.19 & $<0.001$ \\
\hline Muscular thickness, $\mu \mathrm{m}$ & 65.17 & 60.75 & 2.24 & $<0.001$ \\
\hline
\end{tabular}

Table 4

Alpha diversity measures of bacterial communities by 21-day old suckling piglets when feeding maternal diets containing $12 \%$ (LP) or $14 \%$ crude protein (CON). 


\begin{tabular}{|lllll|}
\hline Items & LP & CON & SDM & P-value \\
\hline Chao1 & 218.08 & 208.89 & 33.48 & 0.519 \\
\hline Ace & 216.58 & 205.47 & 33.66 & 0.435 \\
\hline Shannon & 2.72 & 1.67 & 0.68 & $<0.001$ \\
\hline Simpson & 0.16 & 0.45 & 0.13 & 0.001 \\
\hline Coverage & 0.9996 & 0.9996 & $<0.001$ & 0.898 \\
\hline
\end{tabular}

Table 5

Phylum-level taxonomic composition of the jejunal bacterial communities by 21-day old suckling piglets when feeding maternal diets containing $12 \%$ (LP) or $14 \%$ crude protein (CON).

\begin{tabular}{|lllll|}
\hline Phylum & LP & CON & SDM & P-value \\
\hline Firmicutes & 0.51169 & 0.83253 & 0.17449 & 0.002 \\
\hline Proteobacteria & 0.39987 & 0.09948 & 0.15060 & 0.001 \\
\hline Bacteroidetes & 0.02626 & 0.02173 & 0.03188 & 0.299 \\
\hline Chlamydiae & 0.00004 & 0.00804 & 0.01304 & 0.686 \\
\hline Epsilonbacteraeota & 0.01906 & 0.00739 & 0.02340 & 0.166 \\
\hline Cyanobacteria & 0.00210 & 0.00414 & 0.00565 & 0.773 \\
\hline Fusobacteria & 0.00397 & 0.00372 & 0.00485 & 0.525 \\
\hline Actinobacteria & 0.00452 & 0.00332 & 0.00593 & 0.356 \\
\hline Patescibacteria & 0.00176 & 0.00111 & 0.00204 & 0.817 \\
\hline Acidobacteria & 0.00110 & 0.00032 & 0.00140 & 0.840 \\
\hline Tenericutes & 0.00070 & 0.00014 & 0.00112 & 0.544 \\
\hline Cloacimonetes & 0.00009 & 0.00010 & 0.00035 & 0.544 \\
\hline Chloroflexi & 0.00048 & 0.00007 & 0.00072 & 0.312 \\
\hline Verrucomicrobia & 0.00008 & 0.00005 & 0.00020 & 0.356 \\
\hline Planctomycetes & 0.00024 & 0.00002 & 0.00037 & 0.908 \\
\hline Gemmatimonadetes & 0.00022 & 0.00002 & 0.00056 & 0.470 \\
\hline Unknown & 0.02785 & 0.01781 & 0.02738 & 0.156 \\
\hline
\end{tabular}


Table 6

Genus-level taxonomic composition of the jejunal bacterial communities by 21-day old suckling piglets when feeding maternal diets containing $12 \%(\mathrm{LP})$ or $14 \%$ crude protein (CON). 


\begin{tabular}{|c|c|c|c|c|}
\hline Genus & LP & $\mathrm{CON}$ & SDM & P-value \\
\hline Lactobacillus & 0.25881 & 0.76331 & 0.13670 & $<0.001$ \\
\hline Escherichia-Shigella & 0.15483 & 0.02514 & 0.12003 & 0.050 \\
\hline Actinobacillus & 0.12509 & 0.02318 & 0.07921 & 0.050 \\
\hline Buchnera & 0.05169 & 0.01920 & 0.05861 & 0.488 \\
\hline Romboutsia & 0.06841 & 0.01856 & 0.06543 & 0.166 \\
\hline Clostridium_sensu_stricto_1 & 0.09503 & 0.01698 & 0.07304 & 0.003 \\
\hline Acinetobacter & 0.01295 & 0.00957 & 0.01571 & 0.248 \\
\hline Prevotella_7 & 0.00384 & 0.01020 & 0.02064 & 0.436 \\
\hline Chlamydia & 0.00004 & 0.00804 & 0.01298 & 0.686 \\
\hline Helicobacter & 0.01813 & 0.00691 & 0.02292 & 0.094 \\
\hline Veillonella & 0.02581 & 0.00659 & 0.01388 & 0.015 \\
\hline Turicibacter & 0.00703 & 0.00440 & 0.01058 & 0.011 \\
\hline Rickettsia & 0.01763 & 0.00407 & 0.01963 & 0.686 \\
\hline uncultured_bacterium_f_Muribaculaceae & 0.00853 & 0.00352 & 0.00993 & 0.326 \\
\hline Fusobacterium & 0.00326 & 0.00329 & 0.00419 & 0.644 \\
\hline Pseudomonas & 0.00922 & 0.00300 & 0.01422 & 0.106 \\
\hline Terrisporobacter & 0.01388 & 0.00331 & 0.01267 & 0.299 \\
\hline Bacteroides & 0.00514 & 0.00264 & 0.00618 & 0.184 \\
\hline Enterobacter & 0.00117 & 0.00237 & 0.00358 & 0.603 \\
\hline Megasphaera & 0.01073 & 0.00276 & 0.01537 & 0.386 \\
\hline Streptococcus & 0.00261 & 0.00183 & 0.00164 & 0.149 \\
\hline Pasteurella & 0.00642 & 0.00150 & 0.00635 & 0.194 \\
\hline uncultured_bacterium_f_Lachnospiraceae & 0.00161 & 0.00105 & 0.00270 & 0.795 \\
\hline Epulopiscium & 0.00100 & 0.00116 & 0.00153 & 0.225 \\
\hline Citrobacter & 0.00164 & 0.00093 & 0.00206 & 0.453 \\
\hline Prevotellaceae_UCG-001 & 0.00160 & 0.00064 & 0.00226 & 0.149 \\
\hline Lachnoclostridium & 0.00174 & 0.00070 & 0.00185 & 0.100 \\
\hline uncultured_bacterium_f_Clostridiales_vadinBB60_group & 0.00295 & 0.00067 & 0.00352 & 0.260 \\
\hline
\end{tabular}




\begin{tabular}{|lllll|}
\hline Wolbachia & 0.00205 & 0.00058 & 0.00233 & 0.624 \\
\hline Acidaminococcus & 0.00419 & 0.00065 & 0.00800 & 0.386 \\
\hline Sutterella & 0.00240 & 0.00023 & 0.00299 & 0.356 \\
\hline Others & 0.05272 & 0.03520 & 0.01200 & 0.150 \\
\hline Unknown & 0.02785 & 0.01781 & 0.02738 & 0.156 \\
\hline
\end{tabular}

\section{Table 7}

Detailed information of significantly different metabolites at variable importance in projection (VIP) $>1$ and $\mathrm{P}<0.05$ (t-test) by 21 -day old suckling piglets when feeding maternal diets containing $12 \%(\mathrm{LP})$ or $14 \%$ crude protein (CON). 


\begin{tabular}{|c|c|c|c|c|c|c|}
\hline Metabolite name & adduct & $\mathrm{m} / \mathrm{z}$ & $\mathrm{RT}(\mathrm{s})$ & VIP & $\begin{array}{l}\text { FC } \\
\text { (LP/Con) }\end{array}$ & $\begin{array}{l}\mathrm{P} \text { - } \\
\text { value }\end{array}$ \\
\hline Cytosine & $(\mathrm{M}+\mathrm{H})+$ & 112.0496 & 190.3420 & 5.0629 & 1.8512 & 0.0202 \\
\hline Uracil & $(M+H)+$ & 113.0341 & 91.9875 & 3.0508 & 0.8447 & 0.0143 \\
\hline D-Proline & $(M+H)+$ & 116.0704 & 298.3865 & 8.4162 & 1.2016 & 0.0166 \\
\hline L-Valine & $(M+H)+$ & 118.0854 & 287.4770 & 2.7252 & 1.2772 & 0.0099 \\
\hline Methylmalonic acid & $(M+H)+$ & 119.0336 & 168.9700 & 1.3445 & 0.8350 & 0.0033 \\
\hline Nicotinate & $(M+H)+$ & 124.0382 & 203.9075 & 2.8918 & 1.9715 & 0.0351 \\
\hline 5-Methylcytosine & $(\mathrm{M}+\mathrm{H})+$ & 126.0646 & 188.8230 & 1.3633 & 1.6714 & 0.0277 \\
\hline L-Leucine & $(\mathrm{M}+\mathrm{H})+$ & 132.1010 & 252.5760 & 5.5735 & 1.2929 & 0.0046 \\
\hline Dopamine & $\begin{array}{l}(\mathrm{M}+\mathrm{H}- \\
\mathrm{H} 2 \mathrm{O})+\end{array}$ & 136.0744 & 289.8395 & 1.8309 & 1.2324 & 0.0313 \\
\hline Hypoxanthine & $(\mathrm{M}+\mathrm{H})+$ & 137.0451 & 168.9640 & 11.3354 & 0.8197 & 0.0041 \\
\hline DL-O-tyrosine & $\begin{array}{l}(\mathrm{M}+\mathrm{H}- \\
2 \mathrm{H} 2 \mathrm{O})+\end{array}$ & 146.0581 & 249.2860 & 1.0860 & 1.3591 & 0.0089 \\
\hline DL-3-Phenyllactic acid & $\begin{array}{l}(\mathrm{M}+\mathrm{H}- \\
\mathrm{H} 2 \mathrm{O})+\end{array}$ & 149.0577 & 246.7525 & 1.1128 & 1.2927 & 0.0057 \\
\hline L-Methionine & $(\mathrm{M}+\mathrm{H})+$ & 150.0565 & 274.8420 & 2.4280 & 1.2986 & 0.0027 \\
\hline 3-Methyl-L-histidine & $\begin{array}{l}(\mathrm{M}+\mathrm{H}- \\
\mathrm{H} 2 \mathrm{O})+\end{array}$ & 152.0801 & 46.0890 & 3.9454 & 0.3010 & 0.0357 \\
\hline $\begin{array}{l}\text { trans-2-Hydroxycinnamic } \\
\text { acid }\end{array}$ & $(M+H)+$ & 165.0532 & 289.8605 & 1.9537 & 1.2321 & 0.0359 \\
\hline L-Phenylalanine & $(\mathrm{M}+\mathrm{H})+$ & 166.0851 & 246.5560 & 5.2926 & 1.2407 & 0.0113 \\
\hline L-Citrulline & $(\mathrm{M}+\mathrm{H})+$ & 176.1007 & 378.7415 & 1.2356 & 1.3942 & 0.0480 \\
\hline L-Tyrosine & $(\mathrm{M}+\mathrm{H})+$ & 182.0796 & 289.7825 & 2.5042 & 1.2456 & 0.0250 \\
\hline DL-Indole-3-lactic acid & $\begin{array}{l}(\mathrm{M}+\mathrm{H}- \\
\mathrm{H} 2 \mathrm{O})\end{array}$ & 188.0691 & 249.5480 & 3.9166 & 1.3756 & 0.0078 \\
\hline Val-Ala & $(M+H)+$ & 189.1215 & 46.9895 & 2.0810 & 1.8284 & 0.0104 \\
\hline L-Tryptophan & $(\mathrm{M}+\mathrm{H})+$ & 205.0947 & 249.2050 & 2.4176 & 1.4057 & 0.0247 \\
\hline Pantothenate & $(\mathrm{M}+\mathrm{H})+$ & 220.1164 & 257.4480 & 2.2720 & 0.8360 & 0.0199 \\
\hline Val-Leu & $(\mathrm{M}+\mathrm{H})+$ & 231.1675 & 185.4245 & 1.2683 & 1.7742 & 0.0160 \\
\hline Linoleic acid & $\begin{array}{l}(\mathrm{M}+\mathrm{NH} 4) \\
+\end{array}$ & 298.2712 & 42.8735 & 2.1834 & 0.7078 & 0.0268 \\
\hline
\end{tabular}




\begin{tabular}{|c|c|c|c|c|c|c|}
\hline Palmitoyl ethanolamide & $(\mathrm{M}+\mathrm{H})+$ & 300.2855 & 37.4730 & 2.1904 & 0.5074 & 0.0372 \\
\hline Decanoyl-L-carnitine & $M+$ & 316.2439 & 178.3130 & 1.5638 & 2.3734 & 0.0289 \\
\hline$(2 \mathrm{E}, 6 \mathrm{E})$-Farnesol & $\begin{array}{l}(\mathrm{M}- \\
2 \mathrm{H}+3 \mathrm{~K})+\end{array}$ & 337.2477 & 195.5165 & 1.0404 & 3.6265 & 0.0051 \\
\hline Chenodeoxycholate & $\begin{array}{l}(\mathrm{M}+\mathrm{NH} 4) \\
+\end{array}$ & 410.3206 & 144.7795 & 3.5345 & 3.4210 & 0.0292 \\
\hline Cholic acid & $\underset{+}{(\mathrm{M}+\mathrm{NH} 4)}$ & 426.3154 & 210.5310 & 5.4764 & 8.8444 & 0.0092 \\
\hline Glycocholic acid & $(\mathrm{M}+\mathrm{H})+$ & 466.3105 & 227.7410 & 15.0858 & 3.9528 & 0.0033 \\
\hline Tauroursodeoxycholic acid & $\begin{array}{l}(\mathrm{M}+\mathrm{H}- \\
\mathrm{H} 2 \mathrm{O})+\end{array}$ & 482.2866 & 131.6380 & 5.7124 & 5.4911 & 0.0040 \\
\hline Taurochenodeoxycholate & $(\mathrm{M}+\mathrm{H})+$ & 500.2976 & 130.9580 & 11.9799 & 4.7479 & 0.0001 \\
\hline Taurodeoxycholic acid & $\begin{array}{l}(\mathrm{M}+\mathrm{NH} 4) \\
+\end{array}$ & 517.3238 & 131.8080 & 17.7858 & 5.0351 & 0.0004 \\
\hline PC (16:0/16:0) & $(\mathrm{M}+\mathrm{Na})+$ & 756.5424 & 132.4325 & 4.4768 & 0.4513 & 0.0018 \\
\hline Thioetheramide-PC & $(\mathrm{M}+\mathrm{Na})+$ & 758.5586 & 133.0820 & 10.8346 & 0.4940 & 0.0034 \\
\hline Taurocholate & $(2 \mathrm{M}-\mathrm{H})-$ & 1029.5826 & 174.7330 & 2.0868 & 14.2093 & 0.0107 \\
\hline Uracil & $(\mathrm{M}-\mathrm{H})$ - & 111.0207 & 92.4025 & 5.7782 & 0.7934 & 0.0028 \\
\hline L-Leucine & $(\mathrm{M}-\mathrm{H})$ - & 130.0880 & 250.9260 & 6.9081 & 1.3271 & 0.0118 \\
\hline Hydroxyisocaproic acid & $(\mathrm{M}-\mathrm{H})-$ & 131.0711 & 137.5360 & 1.9111 & 2.6679 & 0.0134 \\
\hline Hypoxanthine & $(\mathrm{M}-\mathrm{H})-$ & 135.0321 & 169.3360 & 6.3508 & 0.7611 & 0.0003 \\
\hline 2-Oxoadipic acid & $\begin{array}{l}(\mathrm{M}-\mathrm{H} 2 \mathrm{O}- \\
\mathrm{H})-\end{array}$ & 141.0180 & 365.9160 & 3.1031 & 1.1443 & 0.0390 \\
\hline L-Histidine & $(\mathrm{M}-\mathrm{H})$ - & 154.0621 & 366.8935 & 2.1642 & 1.6983 & 0.0055 \\
\hline L-Phenylalanine & $(\mathrm{M}-\mathrm{H})-$ & 164.0730 & 246.5370 & 5.5839 & 1.3528 & 0.0104 \\
\hline N-Acetyl-DL-methionine & $(\mathrm{M}-\mathrm{H})-$ & 190.0548 & 184.5280 & 1.5021 & 1.7103 & 0.0081 \\
\hline L-Tryptophan & $(\mathrm{M}-\mathrm{H})-$ & 203.0835 & 248.6450 & 2.8622 & 1.4084 & 0.0091 \\
\hline Indoxyl sulfate & $(\mathrm{M}-\mathrm{H})-$ & 212.0031 & 26.7060 & 2.5906 & 0.5467 & 0.0310 \\
\hline $\begin{array}{l}\text { sn-Glycerol 3- } \\
\text { phosphoethanolamine }\end{array}$ & $(\mathrm{M}-\mathrm{H})$ - & 214.0498 & 377.6670 & 1.2265 & 1.3431 & 0.0667 \\
\hline Chenodeoxycholate & $(M-H)-$ & 391.2871 & 144.2480 & 12.7681 & 4.9951 & 0.0373 \\
\hline Cholic acid & $(\mathrm{M}-\mathrm{H})$ - & 407.2823 & 195.3125 & 9.8141 & 4.5789 & 0.0122 \\
\hline \multirow[t]{2}{*}{ Glycochenodeoxycholate } & $(M-H)-$ & 448.3090 & 193.6560 & 5.0633 & 2.1925 & 0.0473 \\
\hline & & Page $25 / 34$ & & & & \\
\hline
\end{tabular}




\begin{tabular}{lllllll}
$\begin{array}{l}\text { 4-Androsten-17.beta.-ol-3- } \\
\text { one glucosiduronate }\end{array}$ & $(\mathrm{M}-\mathrm{H})-$ & 463.2346 & 212.3140 & 1.1474 & 6.7003 & 0.0204 \\
\hline Taurochenodeoxycholate & $(\mathrm{M}-\mathrm{H})-$ & 498.2921 & 132.3305 & 21.3265 & 5.1382 & 0.0001 \\
\hline Glycocholic acid & $(\mathrm{M}-\mathrm{H})-$ & 929.6165 & 227.2290 & 3.0253 & 15.9922 & 0.0194
\end{tabular}

Note. RT, retention time; FC, Fold Change, mean value of peak area obtained from LP/ mean value of peak area obtained from Con.

\section{Figures}


A
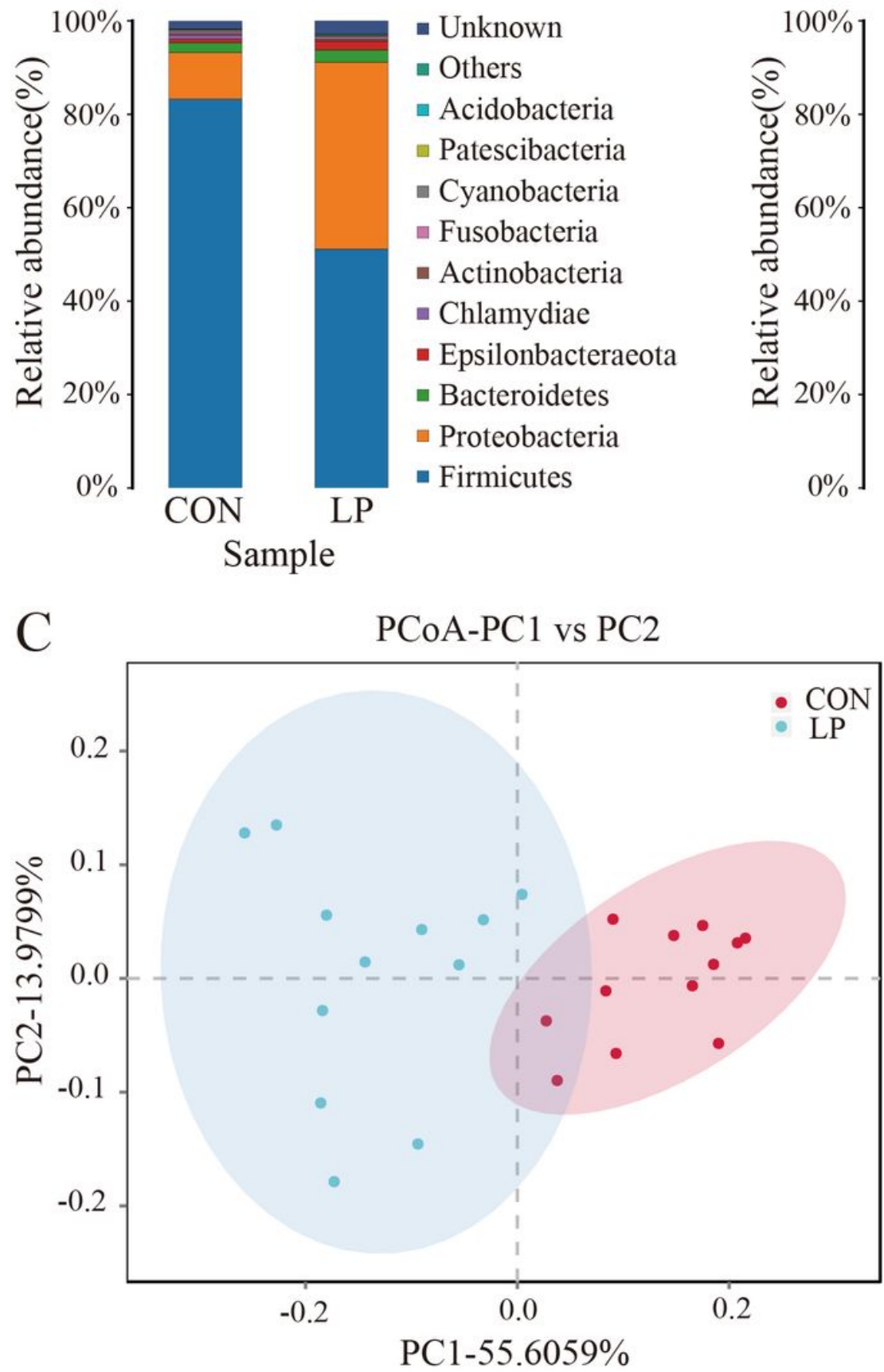

B

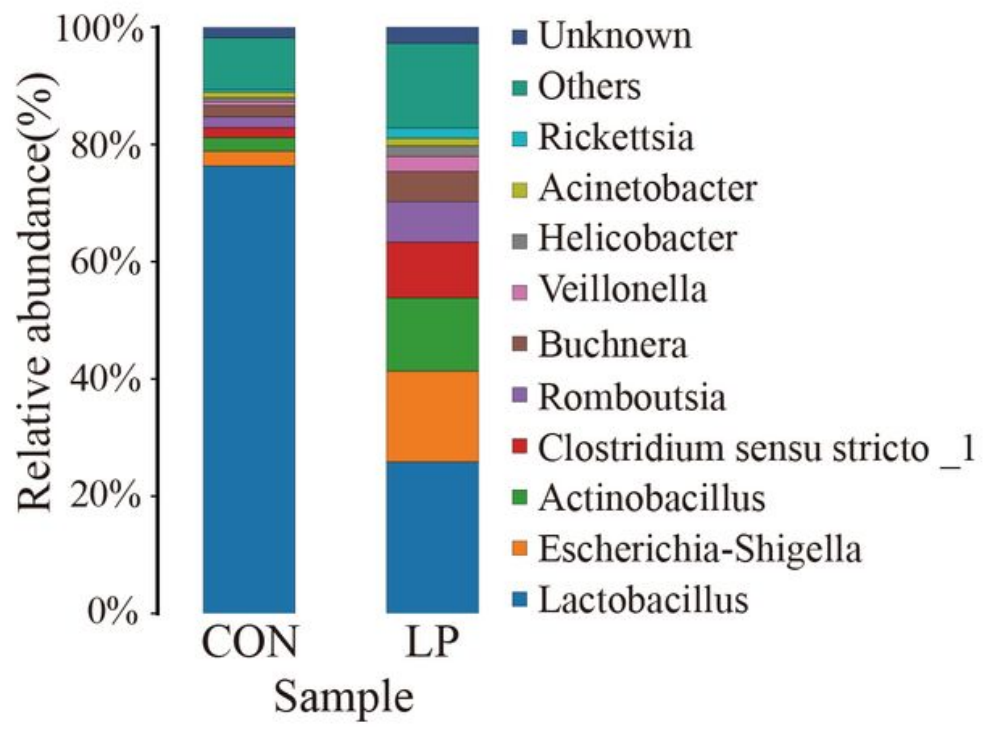

$\mathrm{D}$

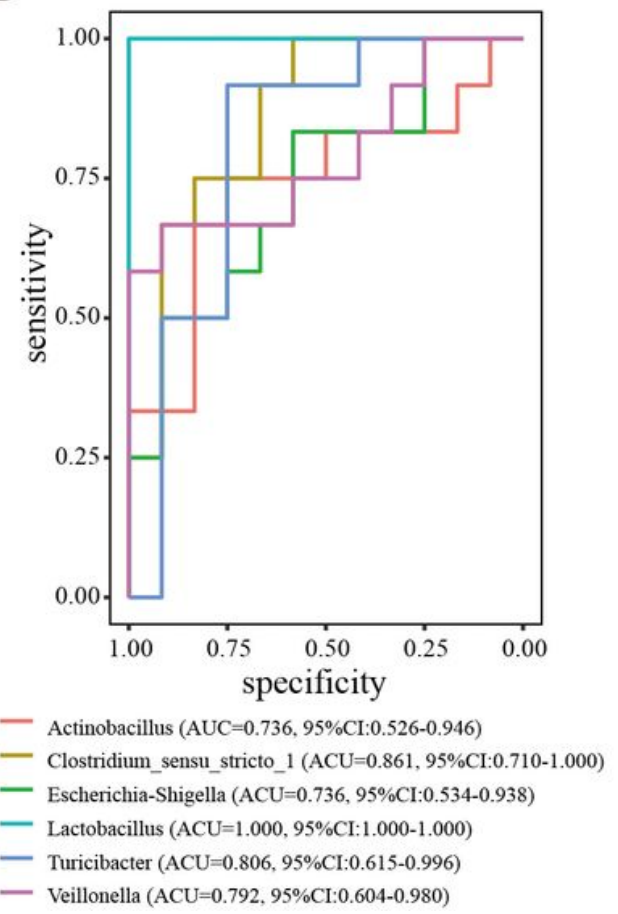

\section{Figure 1}

Classification of the bacterial community composition) by 21-day old suckling Bamei piglets when feeding maternal diets containing $12 \%$ (LP) or $14 \%$ crude protein (CON). (A) Phylum level. (B) genus level. (C) PCoA plot. (D) UPGMA tree. The relative abundance of the top 10 phylum and genus of jejunal microbiome composition profiles revealed by $16 \mathrm{~S}$ rRNA sequencing (each color represents one bacterial). PCoA plot and UPGMA tree using the weighted unifrac similarity method. 
ص CON ש LP

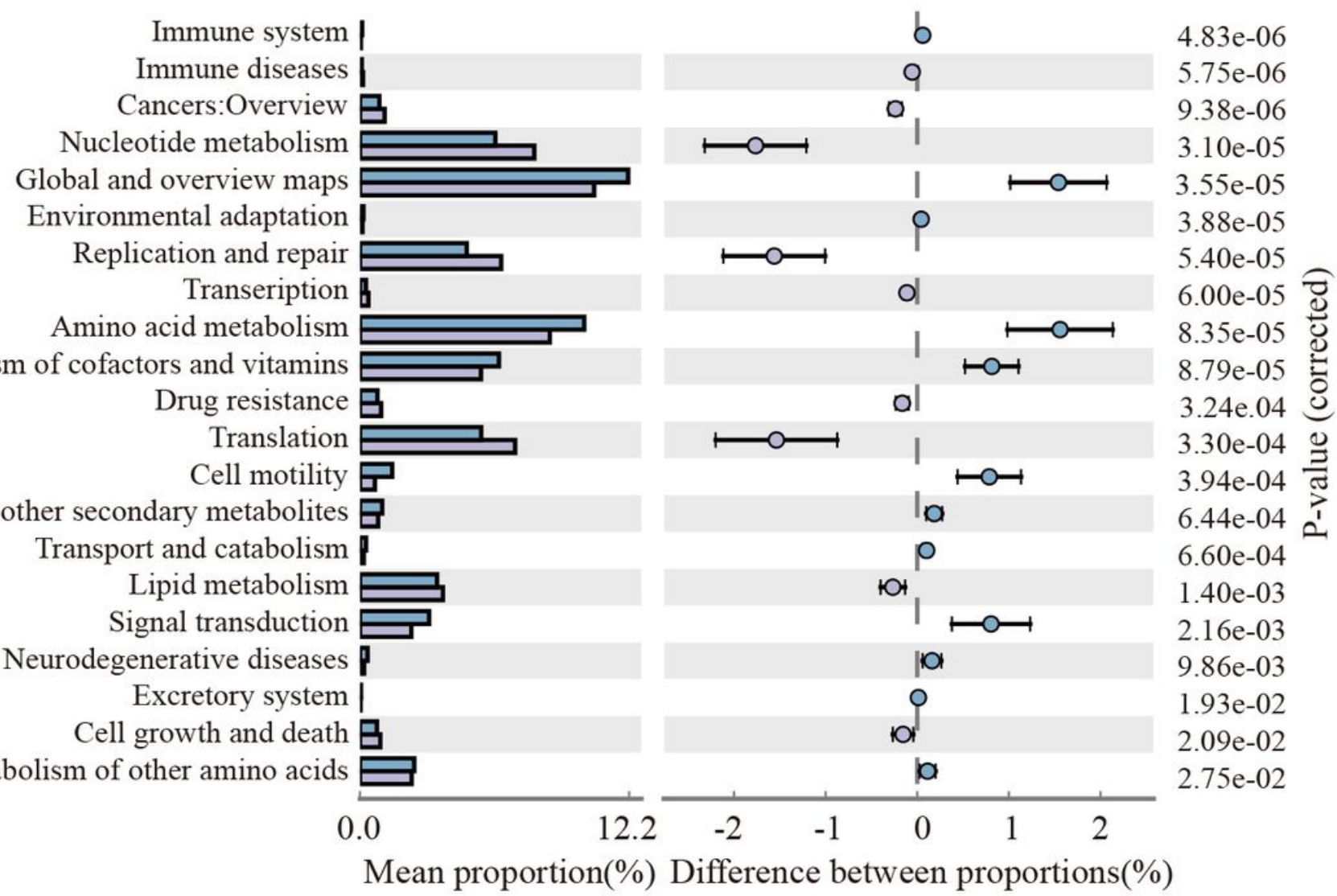

\section{Figure 2}

Predicted microbial functions using PICRUSt by 21-day old suckling piglets when feeding maternal diets containing $12 \%(\mathrm{LP})$ or $14 \%$ crude protein (CON) when bacteria differed. 


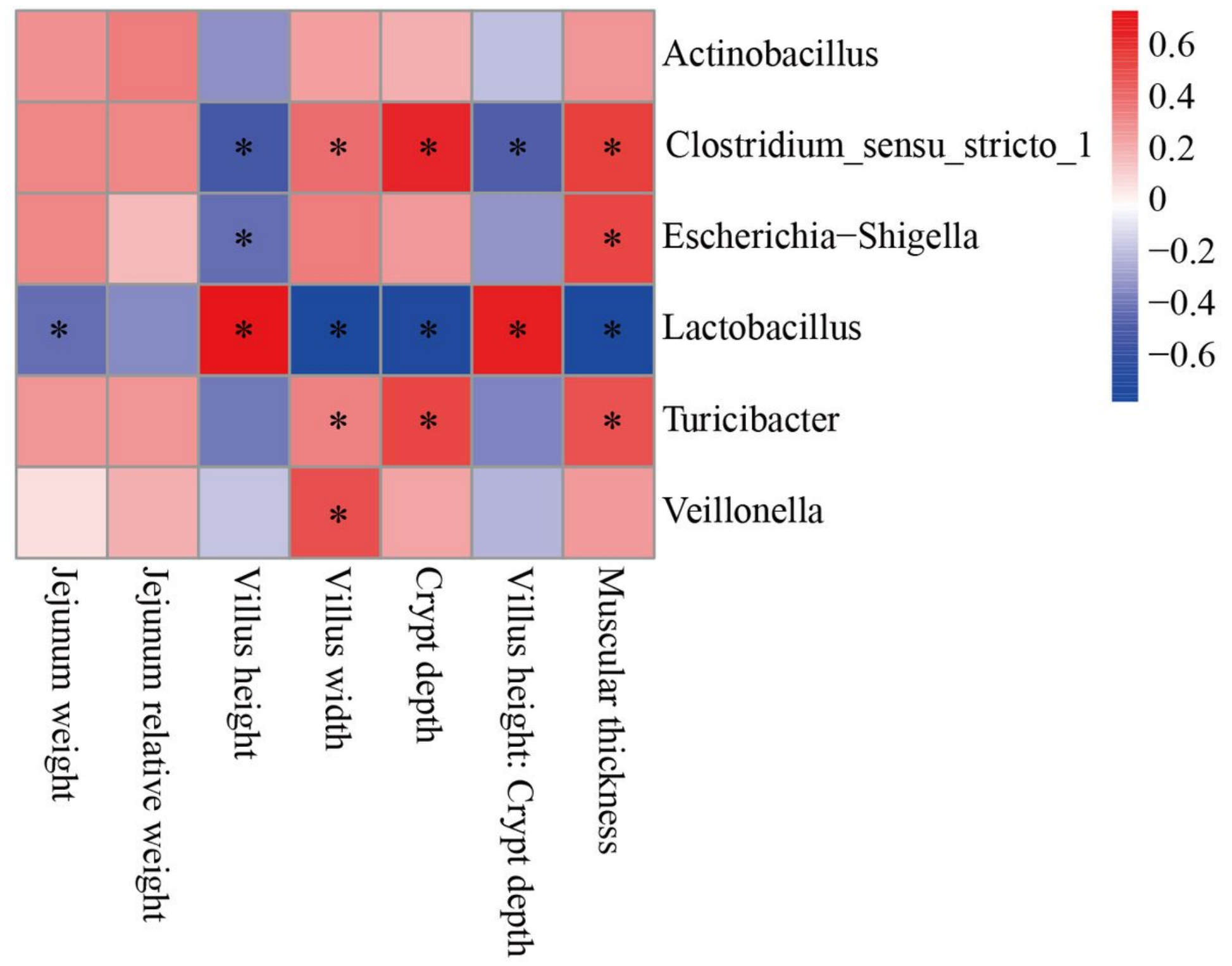

Figure 3

Correlations between differential genera and morphological traits at the jejunum by 21-day old suckling Bamei piglets when feeding maternal diets containing $12 \%(\mathrm{LP} ; \mathrm{N}=12)$ or $14 \%$ crude protein ( $\mathrm{CON} ; \mathrm{N}=12$ ). Each row in the graph represents a genus, each column represents a morphological trait, and each lattice represents a Spearman correlation coefficient between a genus and a morphological trait. Red represents a positive correlation, while blue represents a negative correlation. *Significant correlation between the LP and CON groups $(P<0.05)$. 
A

3D Scores (PCA)

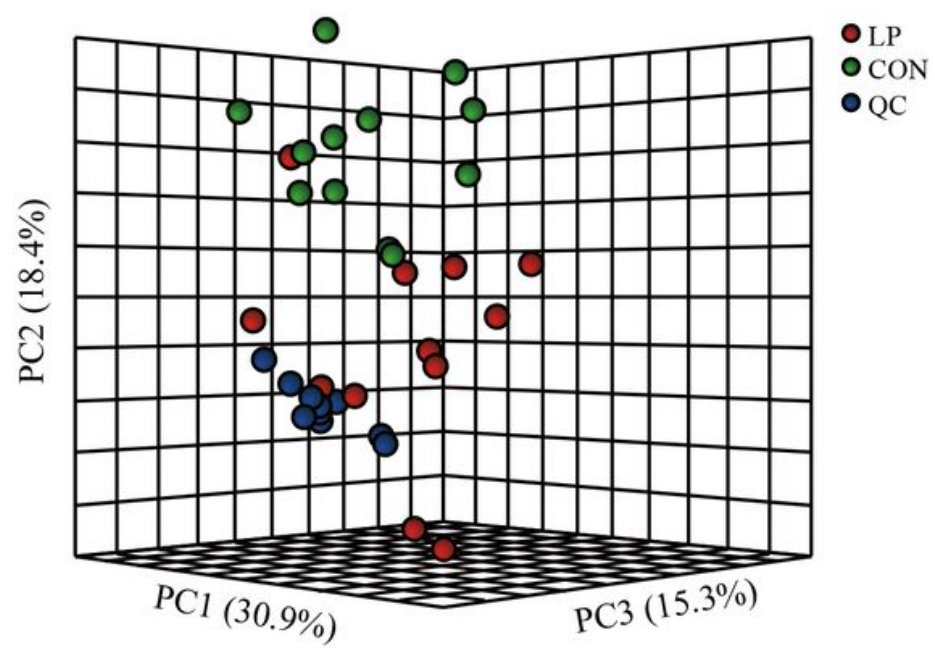

C

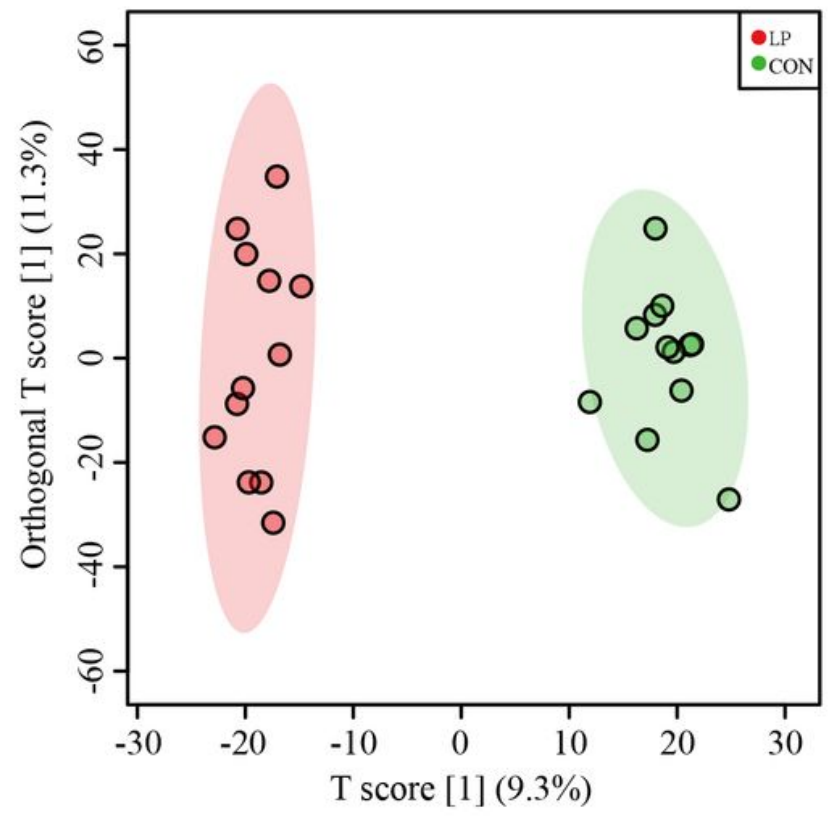

B

3D Scores $(\mathrm{PCA})$

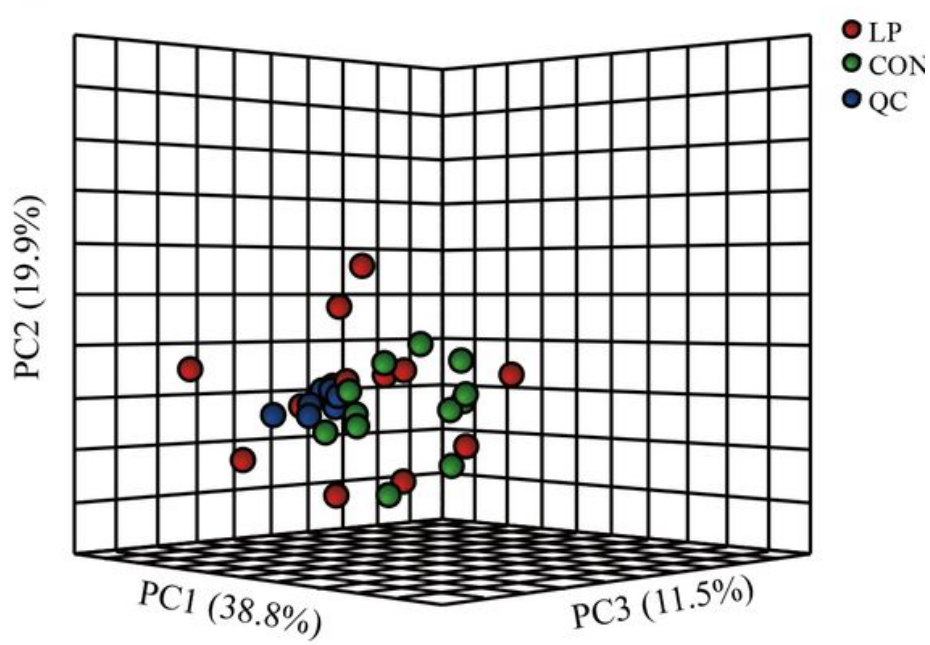

$\mathrm{D}$

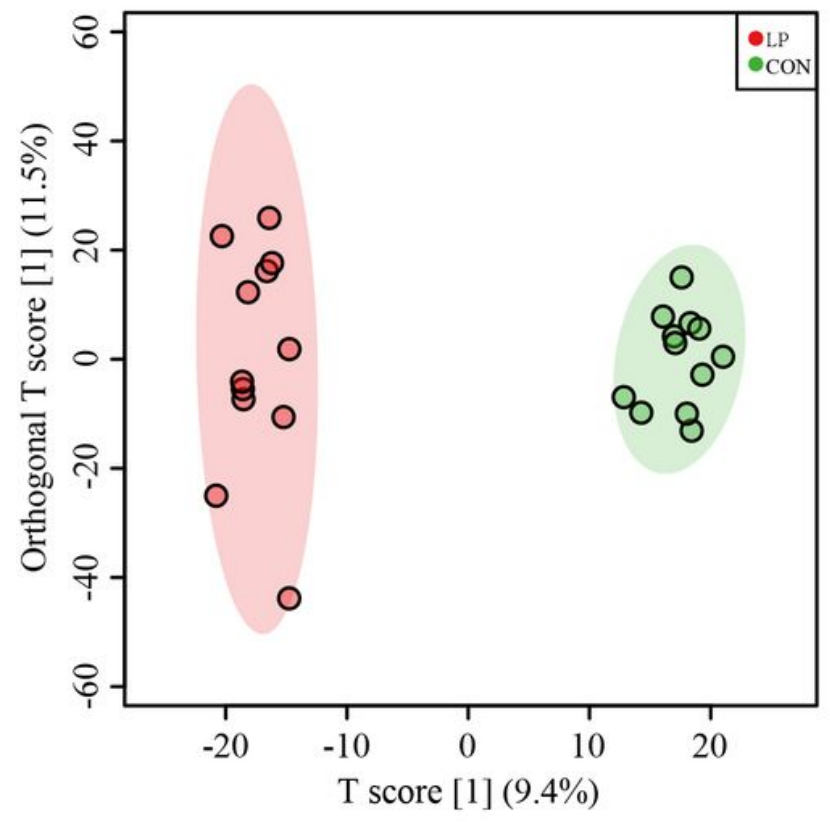

Figure 4

Plot of 3D-PCA and OPLS-DA score, and volcano plot of jejunal metabolites by 21-day old suckling Bamei piglets when feeding maternal diets containing $12 \%$ (LP) or $14 \%$ crude protein (CON) for comparisons following $(A, C, E)$ positive and $(B, D, F)$ negative mode ionization. 


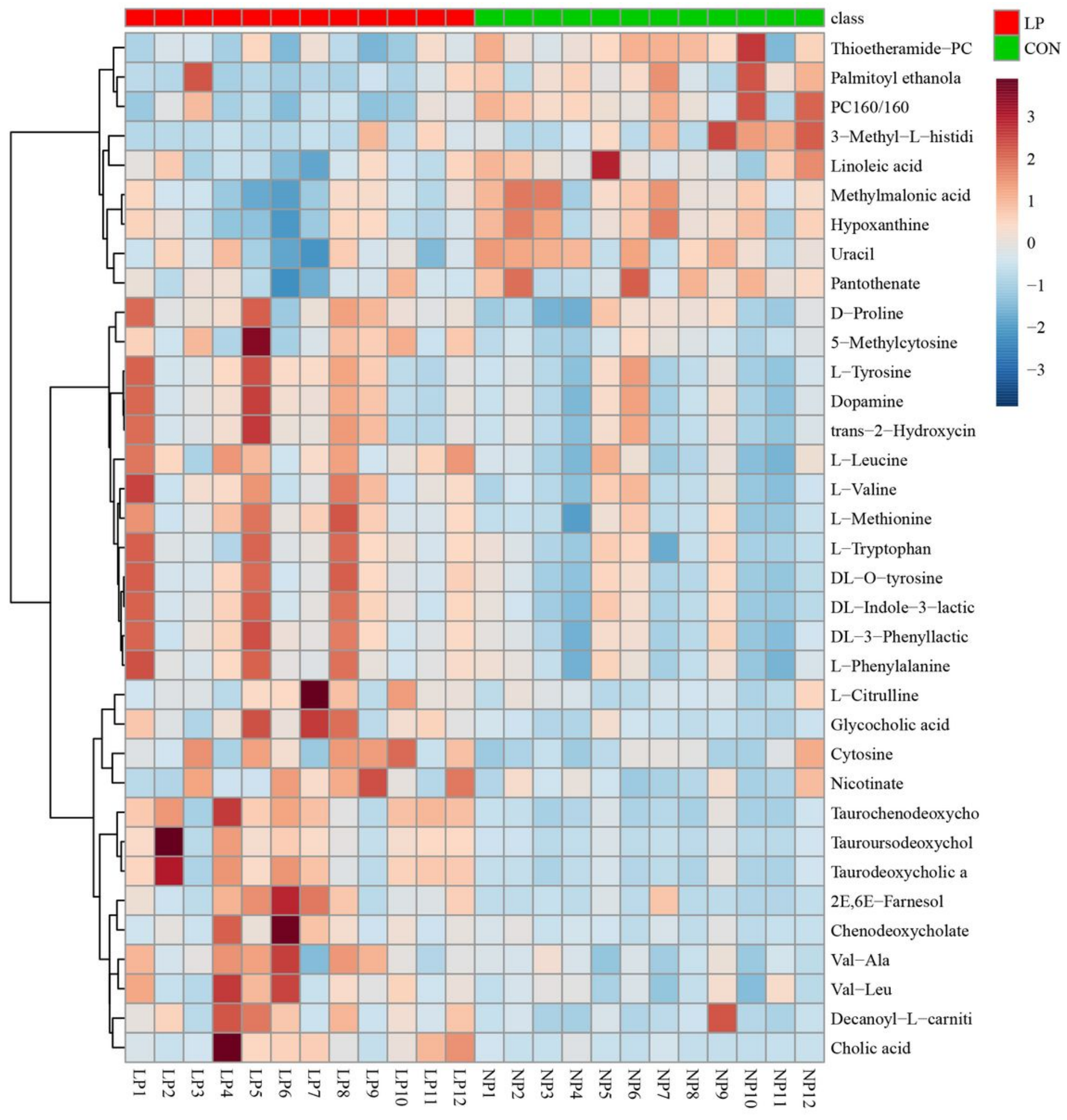

\section{Figure 5}

Hierarchical clustering analysis for identification of different metabolites by 21-day old suckling Bamei piglets when feeding maternal diets containing $12 \%$ (LP) or $14 \%$ crude protein (CON) following positive mode ionization. Each column in the figure represents a sample, each row represents a metabolite, and the color indicates the relative amounts of metabolites expressed in the group; Red indicates that the metabolite is expressed at high levels, and blue indicates lower expression. 


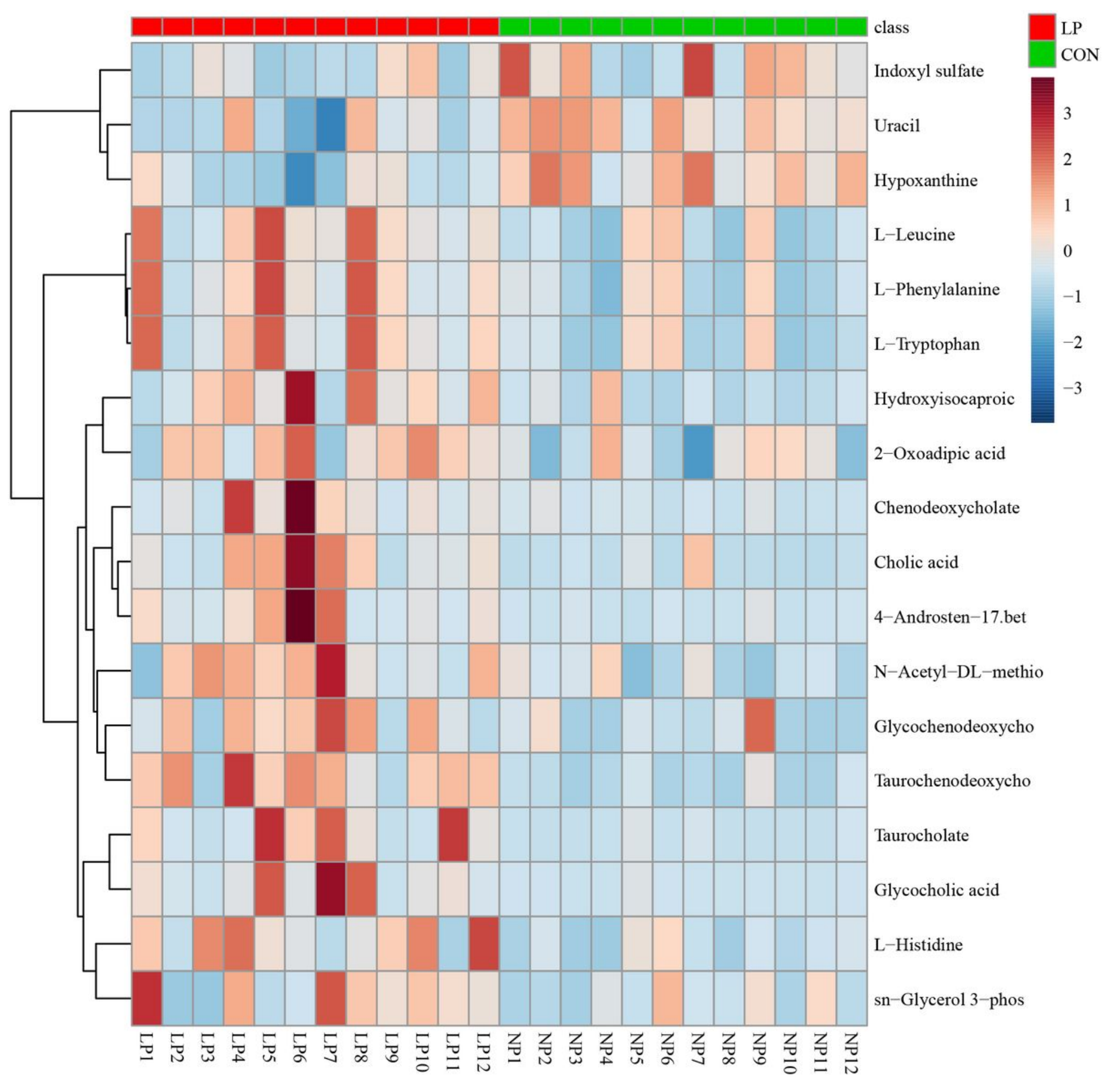

Figure 6

Hierarchical clustering analysis for identification of different metabolites by 21-day old suckling Bamei piglets when feeding maternal diets containing $12 \%$ (LP; red) or $14 \%$ crude protein (CON; green) following negative mode ionization. Each column in the figure represents a sample, each row represents a metabolite, and the color indicates the relative amounts of metabolites expressed in the group; Red indicates that the metabolite is expressed at high levels, and blue indicates lower expression. 
A

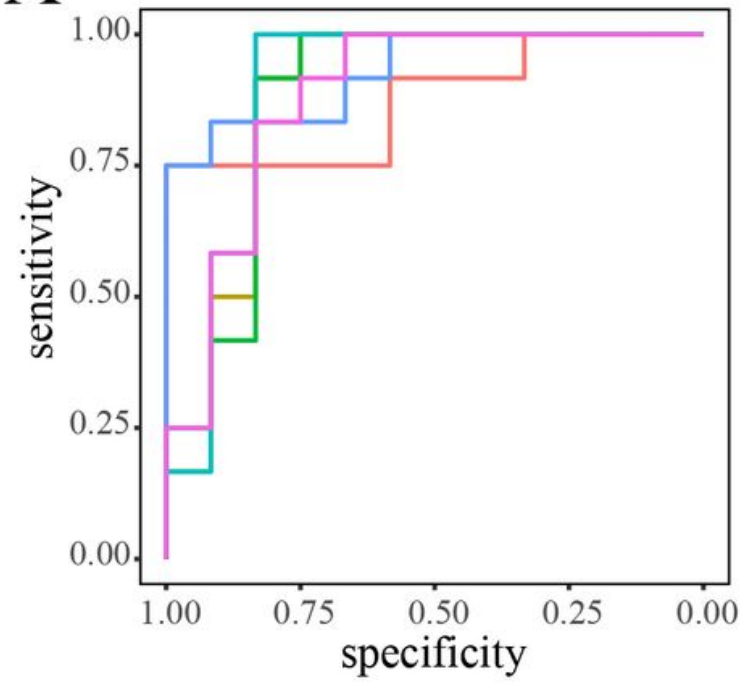

— L-Methionine (AUC $=0.875,95 \% \mathrm{CI}: 0.729-1.000$ )

- Taurochenodeoxycholate (AUC $=0.882,95 \% \mathrm{CI}: 0.729-1.000$ )

- Taurodeoxycholic acid (AUC $=0.875,95 \% \mathrm{CI}: 0.731-1.000$ )

- Tauroursodeoxycholic acid (AUC $=0.896,95 \%$ CI:0.749-1.000)

- 4-Androsten-17.beta. (AUC $=0.931,95 \% \mathrm{CI}: 0.832-1.000$ )

- Cholic acid (AUC $=0.882,95 \% \mathrm{CI}: 0.739-1.000$ )
$\mathrm{B}$

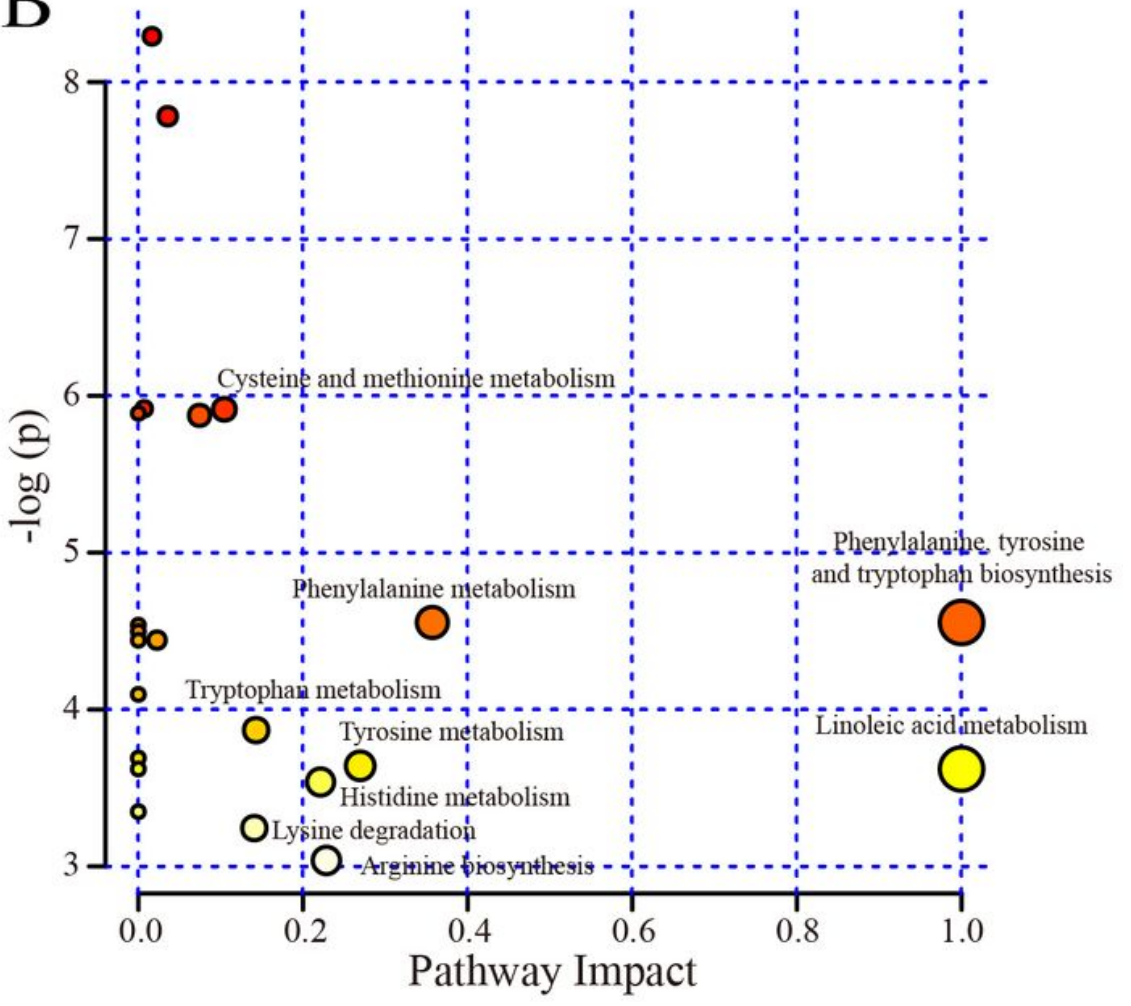

Figure 7

Metabolic pathway enrichment analysis following positive and negative mode ionization to provide metabolite overview by 21-day old suckling Bamei piglets when feeding maternal diets containing $12 \%$ (LP) or $14 \%$ crude protein (CON).

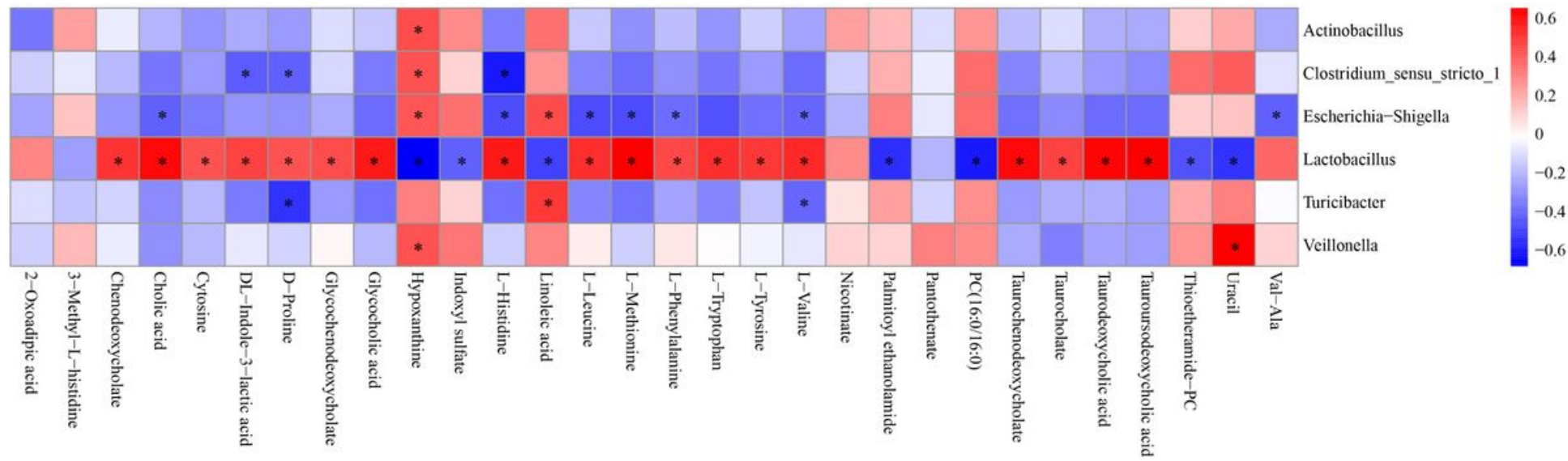

Figure 8

Correlation analysis between genera and metabolite concentrations (VIP $>2$ ) by 21-day old suckling Bamei piglets when feeding maternal diets containing $12 \%$ (LP) or $14 \%$ crude protein (CON). Each row in the graph represents a genus, each column represents a metabolite, and each lattice represents a Pearson correlation coefficient between a component and a metabolite. Red represents a positive correlation, while blue represents a negative correlation. *Significant correlation between the LP and Con groups $(\mathrm{P}<$ 0.05). 
Page 34/34 